\title{
La viticulture antiQue en Provence
}

\author{
Jean-Pierre BRUN
}

Mots-clés. Narbonnaise, Fréjus, Haut-Empire, Antiquité tardive, installations de production, amphores, jarres, tonneaux, pressoirs.

Key-words. Gallia Narbonensis, Fréjus, Early Empire, Late Antiquity, production equipment, amphorae, jars, casks, wine-presses.

Résumé. La viticulture en Narbonnaise orientale est bien connue par les sources antiques, mais la multiplication des fouilles récentes autorise une approche plus déluillée el plus sûre, à la fois des procédés employés el des évolutions. Les recherches archéologiques ont en effet mis au jour des champs de vignes dont certains, à Marseille, remontent à la période hellénistique, et aussi des installations vinicoles, surtout datées du Haut-Empire. Les processus de production et les instruments sont inspirés de modèles italiques : fouloirs à raisin au sol en opus signinum, pressoirs à levier et treuil, - puis à partir du II ${ }^{e}$. à levier et vis -, cuves de recueil du môut, chaudière de cuisson du defrutum et, disposition typique, grands chais équipés de dolia enterrés jusqu'au col.

La viticulture provençale, d'abord limilée à la région de Marseille, puis à la bande côtière, connul un développement considérable après la déduction des colonies d'Arles, Avignon et Fréjus. Puis cet essor s'amplifia et se diversifia pour atteindre son apogée entre la période flavienne et le II' s. Les premiers signes d'une crise agricole, manifestée par un abandon de fermes vinicoles, sont datables de la fin du II s. Par la suite, toutes les installations de production sont progressivement abandonnées dans le courant du III' s. Durant l'Antiquité tardive, bien que notre appréciation soit faussée par l'abandon de la vinification en jarres de terre cuite au profit de tonneaux en bois, il paraît désormais certain que la production de vin n'a jamais regagné l'importance socio-économique qu'elle avait eu sous le. Haut-Empire.

\begin{abstract}
Vine cultivation in the eastern part of Gallia Narbonensis has always been well known from ancient sources. Now with the recent excavations, we have more detailed and precise accounts of both the proceedings in use and the historical developments. Archaeological excavations have revealed vineyards some of which, namely those in Marseille, date back to the Hellenistic period, and installations for wine production date to the Early Empire. Methods of production and implements are influenced by the italic pattern : winepresses standing on a floor made of opus signinum, presses with a lever and axle, then from the $2^{\text {nd }}$ century on, presses with a lever and screw, vintage tubs for the musl, defrutum cooking urns and - a very typical arrangement - big storage rooms with dolia buried in the ground up to the neck.

Vine culture in Provence, first limited to Marseille and its surroundings, then extended to the coastal zone, flourished when the colonies of Arles, Avignon and Fréjus were deducted. This development intensified and reached its peak between the Flavian era and the $2^{\text {nd }}$ century. Viticultural farms started to be abandoned during the $3^{\text {rd }}$ century, as a sign of an approaching agricultural crisis. Despite the fact that the replacement of terracotta jars with wooden barrels might bias our account, it seems quite certain that wine production in Late Antiquity had never again reached the socineconomic significance it had under the Early Empire.
\end{abstract}

La partie de la Narbonnaise située à l'est du Rhône qui constitue la Provence actuelle est une terre accidentée, principalement occupée par des massifs cristallins à l'est et des reliefs calcaires à l'ouest et au nord. Des fleuves - le Rhône, la Durance, l'Argens, le Var - se sont frayé un passage à travers ces masses rocheuses et ont formé des plaines alluviales mais, pour l'essentiel, les terroirs cultivables se situent dans des bassins étroits ceinturés par des collines et drainés par de modestes cours d'eau. Ces terres, caillouteuses, sèches et généralement en pente, sont très favorables aux cultures arbustives et en premier lieu à celle de la vigne qui s'accommode aussi bien des substrats calcaires que cristallins. 
Les sources antiques, relativement abondantes, concernent surtout Marseille et sa chôra: Strabon (Géographie, IV, 1, 2) indique que «le pays est planté d'oliviers et couvert de vignes, mais très pauvre en blé à cause de son sol rocailleux ". Trogue-Pompée évoque un épisode de l'histoire de Marseille au VI ${ }^{\mathrm{c}}$ s. avant J.-C. qui se déroula lors des Anthestéries, fête de la dégustation du vin nouveau (Justin, Abrégé des Philippiques, XLIII, 4, 6). Dans un passage bien connu, il signale aussi que les Massaliotes ont appris la viticulture aux populations indigènes (Justin, XIIIII, 4, 1). Poseidonios, au $\mathrm{II}^{\mathrm{e}} \mathrm{s}$. avant J.-C., rapporte que les Gaulois consomment rituellement du vin importé d'Italie et de Marscille (Athénée, Les Deipnosophisles, IV, 152c-d). À la fin du II ${ }^{\mathrm{e}}$ s. avant J.-C., selon Plutarque (Marius, XXI, 7), les Massaliotes utilisèrent les ossements des Teutons vaincus par Marius pour édifier des murs de clôtures de leurs vignobles. Les Marseillais interdisaicnt à leurs femmes de boire du vin (Elien, Hist. Var., II, 38 ; Athénée, Deipn., X, 429a). Sous l'Empire, leur vin était apprécié et vendu fort cher (Athénée, Deipn., I, 27c). Pline (H. N., XIV, 18) considérait qu'il était le meilleur des Pyrénées aux Alpes et Martial brocarde à quatre reprises le vin de Marseille qu'il accuse d'être vieilli artificiellement à la fumée et de coûter trop cher (Épigrammes, III, 82; X, 36 ; XIII, 123 ; XIV, 108). Quoi qu'il en soit, au II's. après J.-C., le vin de Marseille était apprécié jusqu'en Égypte puisqu'un ostracon de Myos Hormos mentionne onze amphores de vin Massalitanum et, dans une lettre trouvée à Karanis, un correspondant demande qu'on lui apporte des lagènes de vin de Marseille (Bertucchi, 1992, p. 208-209). Pline (H. N., XIV, 68) et Galien (apud Athénćc, I, 27c et Thérapeutique à Glaucon, II, 372) donnent quelques précisions sur la nature de ces vins (voir supra, p. 228 et 234). Au milieu du $\mathrm{V}^{\mathrm{c}} \mathrm{s}$. après J.-C., les vignobles de Marseille faisaient encore la richesse de la cité (Paulin de Pella, Eucharisticos, v. 525-526).

Le reste du vignoble de la Narbonnaise orientale est rarement évoqué. Columelle mentionne à plusieurs reprises le traité viticole de Iulius Graecinus qui était originaire de Forum Iulii et dont un voisin, Paridius Veterensis, était un excellent vigneron (De l'agriculture, IV, $3,6)$. Pline signale que, dans les Alpes-Maritimes, on cultivait une certaine variété de raetica $(H . N$., XIV, 41) et J. André a émis l'hypothèse qu'il s'agissait de plants cultivés autour de Nice et peut-être d'Antibes (1954, p. 365). Mais il faut attendre 739 pour voir mentionné un vignoble précisément localisé : " par testament, Abbon, dernier patrice de Provence, légua à l'abbaye de Novalaise le domaine de Cronia, dans le pagus de Toulon planté en vignes et en oliviers." (Geary, 1985, § 37).

Les recherches archéologiques sur la viticulture ont longtemps souffert de l'attention exclusive apportée aux vestiges de pressoirs tout uniment rapportés à la production de l'huile d'olive. Le comte de Villeneuve, dans la Statistique du département des Bouches-du-Rhône de 1821-1829, publia un pressoir romain à Saint-Jean-deGarguier sans se poser la question de sa destination. Pour Fernand Benoit, à qui l'on doit le commencement des études sur l'archéologic agraire antique en Provence, il ne faisait pas de doutc que tous les pressoirs appartinssent à des huileries qu'il s'agît des installations romaines de la région d'Arles (Benoit, 1936) ou préromaines d'Entremont (Benoit, 1968). Dans mon ouvrage sur L'oléiculture antique en Provence (1986), j'ai aussi attribué la majorité des installations de pressurage à l'huile alors que je savais qu'il est difficile de distinguer les installations oléicoles des vinicoles à partir de prospections (Brun, 1986, p. 223-227). Les recherches menées sur les ateliers d'amphores à Marscille, à Velaux, à Puyloubier (Bouches-du-Rhône), à La Crau, au Castellet, à Sanarysur-Mer, à Fréjus (Var), à Saint-Martin-les-Eaux (Alpes-deHaute-Provence), pour ne citer que quelques exemples, montraient le nombre, l'importance et la vaste distribution géographique de ces officines, et donc de la production de vin, entre le $\mathrm{I}^{\text {cr }}$ et le III $^{e}$ s. de notre ère (Laubenheimer, 1985, p. 81-97, 121-127 et 193-209; 1989a, p. 106-114). Depuis une quinzaine d'années, il s'est avéré que telle ou telle villa produisait surtout du vin. Désormais, des critères de distinction mieux établis (présence de fouloir ou de moulin, découverte de pépins de raisin ou de noyaux d'olives, existence de nombreux dolia, etc.) permettent d'attribuer plus sûrement à l'huile ou au vin les installations fouillées ou prospectées, pour peu qu'un ou plusieurs de ces critères soient observables (Brun, 1993b). Avec les fouilles du Grand Loou à La Roquebrussanne (1981-1983), de Pardigon 3 à Cavalaire-sur-Mer (1985-1986), de Pardigon 2 à La CroixValmer (1984-1991), de la rue du Port à Cavalaire-surMer (1993) et des Toulons à Rians (1993-1996), l'ampleur du phénomène viticole, minoré jusqu'alors, devint manifeste (Brun, 1993a). Depuis lors, les recherches occasionnées par les fouilles d'urgence ont, chaque année, apporté des données nouvelles tant sur les 


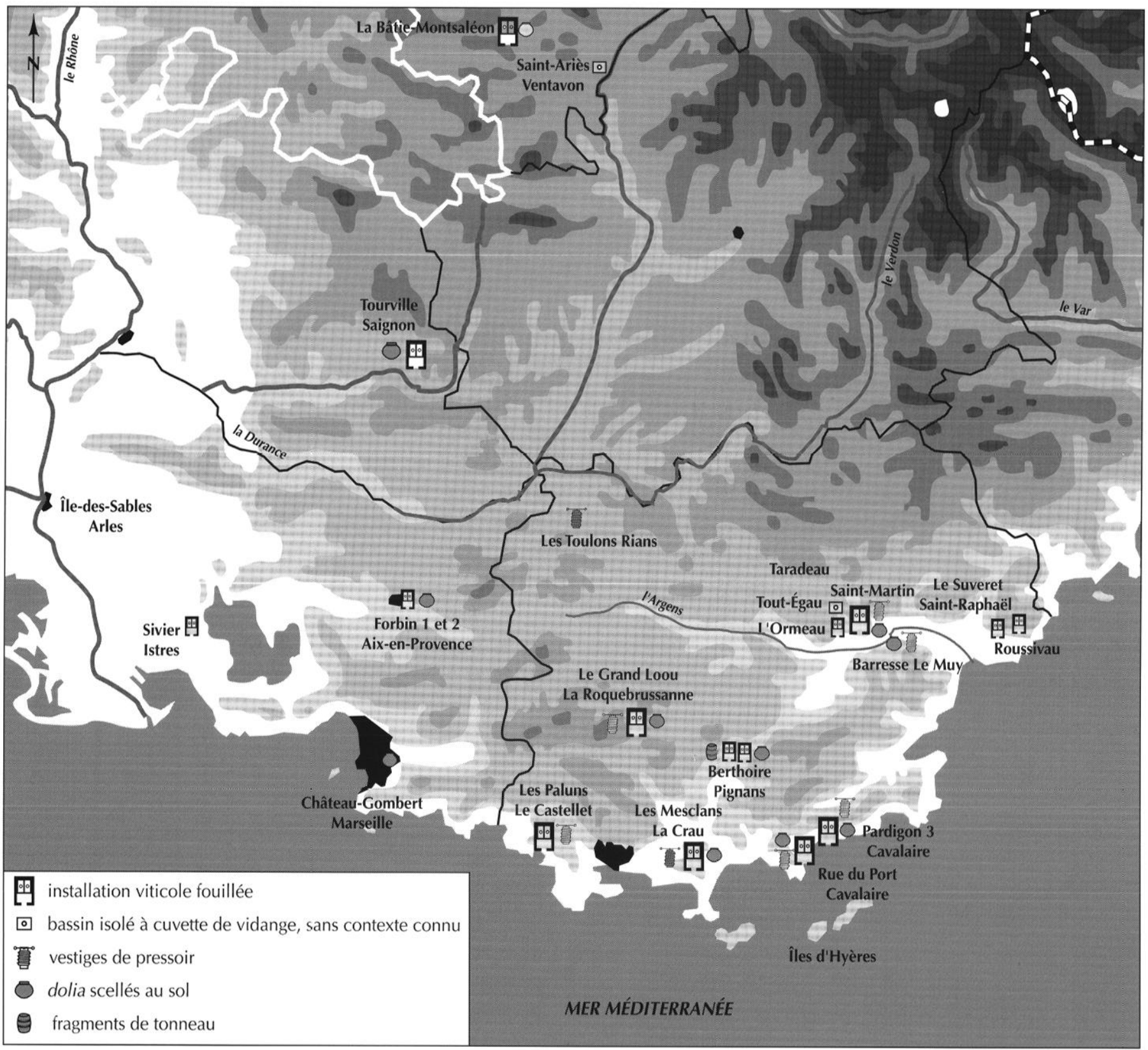

Fig. 22 - Carte des inslallations vinicoles antiques fouillées en Provence.

champs de vignes que sur les installations elles-mêmes (Boissinot, 1994, 1995a ; Brun, 1998) (fig. 22).

\section{LA VITICULTURE PRÉROMAINE}

Selon Aristote (apud Athénée, XII, 576), c'est une coupe de vin mêlé d'eau que présenta Petta, la fille du roi indigène, à Euxénos, le fondateur de Marseille. F. Benoit pensait que ce passage indiquait l'existence d'un commerce du vin rhodien que d'autres, depuis, ont attribué aux Étrusques. Peut-on en tirer argument pour imaginer une viticulture précoloniale? Personne ne risque cette hypothèse malgré la présence de pépins de raisin dans des niveaux de l'âge du Bronze qui proviendraient d'ailleurs de vignes sauvages (voir L. Bouby et P. Marinval, supra, p. 22). Il faut souligner que produire du vin demande un savoir-faire que ne possédaient 
probablement pas les populations de l'âge du Bronze et du début de l'âge du Fer, sinon on s'expliquerait mal leur besoin de boissons alcoolisées, cette " soif celtique » qui était un lieu commun des Anciens.

Dès l'implantation des Phocéens à Marseille, la vigne fut largement plantée dans les alentours de la ville. À partir du milieu du siècle, les débuts de la production d'amphores du type "ionio-massaliète » (Bertucchi, 1992, type 1) marquent l'essor de la production de vin. Les lieux de découverte de ces amphores montrent la diffusion du vin de Marseille sur les rivages de la Provence et du Languedoc et le long de la vallée du Rhône durant les $\mathrm{VI}^{\mathrm{c}}$ et $\mathrm{V}^{\mathrm{c}} \mathrm{s}$. avant J.-C. (Bats dir., 1990, passim). Jusqu'à présent toutefois, on n'a pas trouvé d'installation viticole grecque à Marseille ou dans ses environs. En revanche, $P$. Boissinot a pu identifier des tranchées de culture de vignes d'époque hellénistique à Saint-Jean-duDésert à l'est de la ville (voir supra, p. 55-58). Il est vraisemblable que dans les alentours des forteresses que Marseille avait implantées le long de la côte (Tauroeis, Olbia, Antipolis et Nikaia) les colons grecs ont dû cultiver des oliviers et des vignes, surtout après la division de la chôra en lots individuels ${ }^{117}$ mais force est de constater que, jusqu'à présent, les niveaux classiques et hellénistiques d'Olbia n'ont livré aucun indice allant en ce sens.

Une certaine forme de viticulture s'est répandue dès le V $\mathrm{V}$. chez les indigènes de l'étang de Berre, peut-être sous le contrôle de Marseille. À Martigues, les fouilles de J. Chausserie-Laprée ont mis au jour des amas de pépins de raisin, mais sont-ce les résidus du pressurage des grappes pour faire du vin ou de raisins secs (Jacob et al., 1990, p. 177) ? Sur la rive nord de l'étang de Berre, le site de Coudounèu (Lançon-Provence) a également livré de nombreux pépins de raisin datables de la seconde moitié du IV's. avant J.-C. (Verdin, 1996-1997, p. 189-192). Qu'il s'agisse d'une ferme fortifiée appartenant à un chef local selon l'hypothèse de Patrice Arcelin (1999, p. 465-467), d'un grenier collectif selon celle de Florence Verdin ou d'un simple hameau, on est frappé par le grand nombre des jarres (plus de 150) dont une part a pu servir à la vinification, à moins que, là aussi, les habitants aient entreposé des raisins secs (voir M. Py et R. Buxó

117. J. Benoit (1985) a mis en évidence les traces d'un cadastre grec probablement antérieur à la conquête romaine au nord et à l'est d'Olbia. G. Chouquer et F. Favory (1991, p. 148-151) proposent d'en restituer un autre autour de Tauroeis. i Capdevila, supra, p. 35). Il existait peut-être un pressoir à levier et poids de pierre qui aurait fonctionné dès les débuts de l'occupation, mais rien ne prouve qu'il s'agisse d'un pressoir à vin, sauf l'absence apparente de noyaux d'olives qui paraît exclure l'huile. Plus tard, à la fin du $\mathrm{II}^{\mathrm{e}} \mathrm{s}$. ou au début du $\mathrm{I}^{\mathrm{er}} \mathrm{s}$., en sus de l'huile, la production du vin est attestée à Entremont (fouilles de P. Arcelin dans l'îlot VIII, cf. Gallia Informations, 1990, 1-2, p. 130-131).

Que ces productions aient laissé comme traces surtout des pépins de raisin carbonisés ne doit pas surprendre. Les instruments utilisés étaient le plus souvent en matériaux périssables (fouloir en bois, pressoirs à torsion) et la vinification devait être conduite dans des jarres polyvalentes qu'il est difficile d'identifier formellement. En tout état de cause, la viticulture celtoligure, limitée au pourtour de l'étang de Berre et à quelques terroirs lc long de la côte, ne devait guère concurrencer celle des Marseillais qui bénéficiaient d'un savoir-faire séculaire. Elle ne suffisait pas à couvrir les besoins des populations qui continuaient à acheter du vin marseillais, puis, à partir du III ${ }^{\mathrm{c}}$ s., surtout du vin italien.

$\mathrm{Au} \mathrm{I}{ }^{\mathrm{er}} \mathrm{s}$. avant J.-C., les bouleversements fonciers liés à la guerre civile et aux déductions des colonies entraînèrent un changement majeur dans le mode d'habitat: presque tous les oppida furent progressivement abandonnés et les agriculteurs italiens et indigènes s'établirent en plaine, au milieu des exploitations. Les niveaux archéologiques correspondant à cette période, le plus souvent enfouis sous des habitations postérieures, sont mal conservés et n'ont jusqu'à présent livré aucun vestige d'installation de production du vin.

\section{LA VITICULTURE SOUS LE HAUT-EMPIRE}

Fn dehors du territoire de Marseille, la déduction des colonies entraîna une extension considérable des surfaces consacrées à la vigne. Les colons venus d'Italie apportèrent certainement des plants et leur connaissance de cette culture rémunératrice. On commence à percevoir les débuts de cette expansion grâce aux traces de plantation de vignes aux Dèves et à Lapalud (voir supra, p. 59-60) et aux ateliers d'amphores imitant des formes italiques et espagnoles à Saint-Just (Ardèche), à Mazan et à Sainte-Cécile-les-Vignes (Vaucluse) qui attestent la production et la commercialisation de vin dès 
l'époque augustéenne, au moins dans la colonie d'Orange (Laubenheimer, 1998a, p. 167). Mais les premières infrastructures de vinification sont datables seulement de la fin du I ${ }^{\mathrm{er}}$ s. avant J.-C. et surtout du début $\mathrm{du} \mathrm{I}{ }^{\mathrm{er}} \mathrm{s}$. après J.-C. : le plus ancien indice provient de la périphérie d'Arles, dans l'île des Sables, où, lors de fouilles d'urgence réalisées en 1990, une cuve en chêne datable de cette période a livré un amas de pépins de raisin, probablement un résidu de foulage ${ }^{118}$.

Avant de tirer des conclusions d'ensemble, il convient de présenter quelques exemples de gisements illustrant les divers types d'installations vinicoles.

\section{LES INSTALLATIONS SITUÉES DANS DES AGGLOMÉRATIONS}

Alors qu'à la fin de la Protohistoire, le vin et l'huile semblent avoir surtout été produits dans les oppida, les installations agricoles trouvées dans les agglomérations de l'époque romaine sont rares. C'est à peine si l'on peut citer une huilerie à Glanum et un chai à l'intérieur de la ville d'Aix-en-Provence, mais, dans ce dernier cas, il pourrait s'agir d'un cellier appartenant à un commerçant plutôt qu'à un producteur ${ }^{119}$. Toutefois, les bourgs ruraux devaient regrouper des artisans et de petits agriculteurs cultivant des vignes et des oliviers. Des pressoirs ont été trouvés dans une agglomération romaine partiellement fouillée à Pignans (Var), dans celle de Forum Voconii (Le Cannet-des-Maures, Var) et probablement dans celle de La Bâtie-Montsaléon (Hautes-Alpes).

\section{La Bâtie-Montsaléon (Hautes-Alpes)}

La Bâtie-Montsaléon occupe l'emplacement d'une agglomération secondaire située sur la via Domitia et nommée Mons Seleucus. Le site, connu depuis longtemps, fit l'objet de fouilles étendues en 1804-1805 sous l'impulsion de J.-C.-F. Ladoucette, premier préfet des HautesAlpes. Des habitations, des thermes publics et des rues à

118. Renseignement inédit de Muriel Vecchione que je remercie.

119. Lors des fouilles du boulevard de la République, un chai de plus de $40 \mathrm{~m}$ de long par 9,50 m de large a été mis au jour, mais les structures adjacentes n'ont pas été fouillées. On ne sait donc s'il s'agit d'une partie d'installation agricole ou d'un chai de négociant (Nibodeau et al., 1989, p. 10-11). portiques furent alors dégagés (Ganet, 1995, p. 68-69). Plus tard, en 1836, C. Romieu dégagea, à $40 \mathrm{~m}$ au nord de la mairie de La Bâtie-Montsaléon, un bâtiment de quatre pièces qui semble correspondre à une installation vinicole complète. La description de Ladoucette mérite d'être citée : "dans la partie du levant est une chambre où se trouve neuf grandes urnes incrustées dans un mastic très solide et à peu près toutes de la même dimension, c'est-à-dire 5 pieds de profondeur par 4,5 de largeur. Au milieu de cette chambre est un carré de malonnage si bien conservé qu'on aurait de la peine à l'entamer avec un pic. À côté est une autre chambre avec un glacis bien uni et très solide. Au couchant, on a découvert une chambre où sont encore cinq grandes urnes mais elles ne sont pas aussi bien conservées que les autres. Au milieu de ce même bâtiment, on a trouvé trois grandes pierres taillées d'une longueur de $13 \mathrm{~m}$, creusées pour servir à un écoulement quelconque. Ce bâtiment, rasé à fleur de terre comme les constructions trouvées de 1804 à 1805 , renfermait 14 urnes dont sept apparurent brisées. Ces urnes ou amphores étaient en terre cuite, éloignées les unes des autres d'un mètre environ et disposées sur trois lignes. On prétend que plusieurs d'entre elles avaient une telle capacité qu'elles pouvaient contenir chacune deux personnes : en effet, leurs dimensions étaient d'un mètre de diamètre et de $1,50 \mathrm{~m}$ de profondeur. Elles se trouvaient enchâssées dans un mastic rougeâtre et poli, si dur qu'il ne cédait qu'avec peine à la hache. Un petit conduit, dont les bords faits en même mastic s'élevaient à $3 \mathrm{~cm}$ en dessus du sol régnait de l'une à l'autre. " (Ladoucette, 1848, p. 343-344). On doit vraisemblablement reconnaître dans cet ensemble une cuve en béton de tuileau (" mastic rougeâtre »), un chai contenant 9 dolia defossa reliés entre eux et à la cuve par un système de canaux de remplissage et un autre chai plus petit abritant 5 dolia. Il est possible que les pierres taillées comportant un écoulement aient appartenu à un pressoir (contrepoids avec rainure à leur face supérieure ou canalisation en pierre ?). Les conditions de fouilles de l'époque ne permettent pas, bien entendu, de préciser la datation de ces structures, mais l'essentiel du mobilier trouvé est datable des $\mathrm{I}^{\text {er }}$ et $\mathrm{II}^{\mathrm{e}} \mathrm{s}$. après J.-C.

\section{Berthoire à Pignans (Var)}

En 1997, une fouille d'urgence menée par M. Borréani du Centre archéologique du Var au lieu-dit 


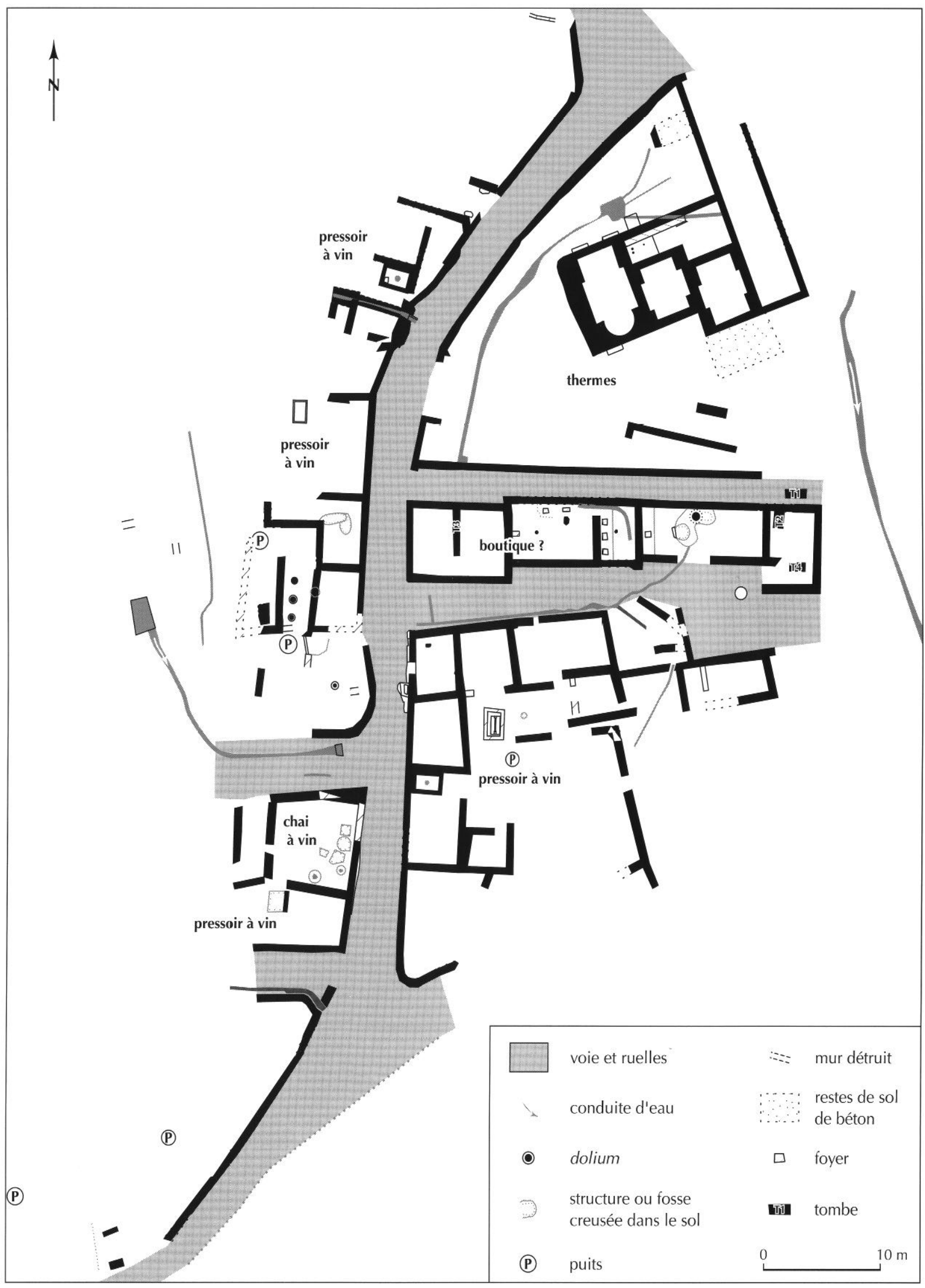

Fig. 23 - Plan d'un quartier de l'agglomération secondaire de Berthoire à Pignans, Var (plan F. Laurier, Centre archéologique du Var). 


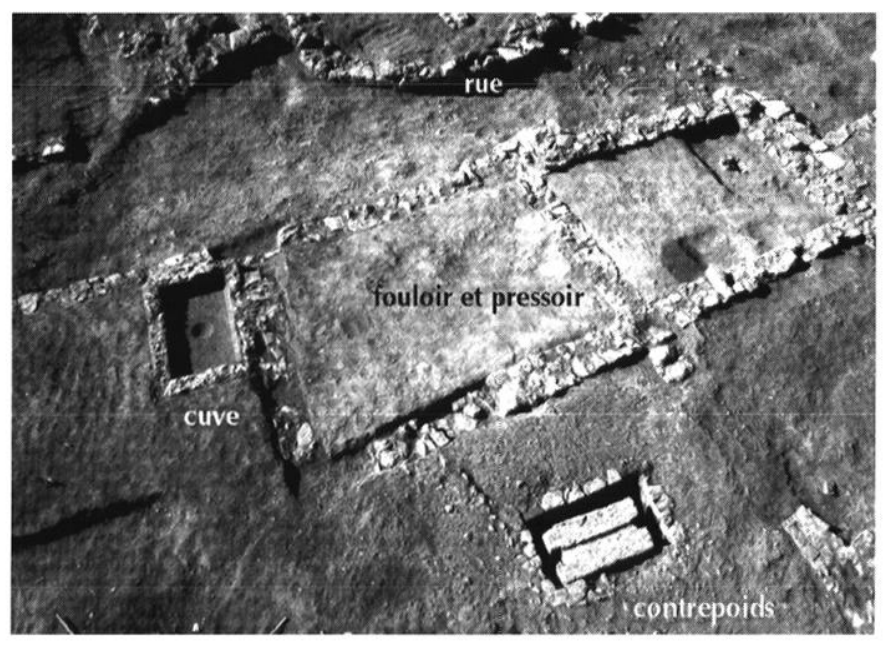

Fig. 24 - Pressoir el cuve à vin de Berthoire à Pignans, Var (cliche.J.-P. Brun).

Berthoire, à la sortie ouest du village, a permis de dégager un quartier d'habitat de l'époque romaine (Brun, 1999, p. 556-560). Outre de petits thermes publics et des boutiques, cinq habitations étaient implantées de part et d'autre d'une voie secondaire reliant Forum Voconii à Telo Martius (Toulon) (fig. 23). Construites à la fin du I ${ }^{\mathrm{er}} \mathrm{s}$. après J.-C. et occupées jusque vers le milieu du III $^{\mathrm{e}} \mathrm{s}$., toutes comportaient des structures de production de vin. Malgré l'arasement des vestiges, on a pu déterminer qu'il s'agissait de petites unités possédant un fouloir combiné avec un pressoir à levier, une cuve bétonnée et des celliers abritant des dolia (fig. 24). Les leviers des pressoirs étaient manœuvrés par des câbles tirés par des treuils fixés sur des contrepoids de pierre et placés dans des fosses quadrangulaires. La production de chaque viticulteur était peu importante : l'un n'avait que trois dolia (soit une capacité de l'ordre de $45 \mathrm{hl}$ au maximum), un autre une dizaine (soit 100 à $150 \mathrm{hl}$ ). Mais ces chiffres sont peut-être à majorer si, notamment au III ${ }^{\mathrm{c}}$ s., on utilisait des tonneaux non seulement pour le transport mais aussi pour la vinification : un fragment de couvercle de tonneau a été trouvé dans un puits comblé dans la première moitié du III's $s$.

\section{Forum Voconii, Le Cannet-des-Maures (Var)}

Plusieurs blocs de pressoirs étaient connus depuis longtemps sur ce site et des fouilles effectuées en 2000 par G. Congès et $F$. Martos ont mis au jour, à l'intérieur de l'agglomération, un pressoir daté du $\mathrm{II}^{\mathrm{e}} \mathrm{s}$. L'instal- lation n'est toutefois pas assez complète pour déterminer s'il s'agit d'un pressoir à vin ou à huile.

\section{LES INSTALLATIONS DOMANIALES}

Pour l'essentiel, le vin était produit dans les exploitations agricoles dispersées dans la campagne. Les installations domaniales peuvent être réparties en deux groupes : les chais comportant un équipement minimal (un fouloir et une cuve) et les grands domaines dotés de plusieurs fouloirs et pressoirs.

La première catégorie, qui commence à être bien représentée, correspond à des villae modestes, parfois à des fermes qui semblent avoir été occupées par des descendants des populations indigènes. Ces exploitations devaient pratiquer une large polyculture associée à un petit élevage. Les quelques exemples qui suivent paraissent éclairants.

\section{$1^{\text {er }}$ GROUPE : INSTALLATIONS DE BASE}

\section{Barresse au Muy (Var)}

La ferme de Barresse, située en limite nord du massif des Maures, a été fouillée par Jacques Bérato en 1995 (Brun, 1999, p. 533-535). Les bâtiments, établis sur quatre plans, forment un quadrilatère hors d'œuvre d'environ $11 \mathrm{~m}$ de large sur $23 \mathrm{~m}$ de long (fig. 25). Leur occupation paraît n'avoir pas dépassé un siècle, entre les années 150 et 250 au plus. Les pièces, au sol en terre, étaient destinées à l'habitation ( $\mathrm{n}^{\text {\% }} 4$ et 6$)$, à la cuisson des poteries ( $\mathrm{n}^{\mathrm{os}} 8$ et 9 ) et à l'élaboration du vin $\left(n^{o s} 1,2\right.$ et 5$)$. Une pièce pratiquement carrée $\left(n^{\circ} 1\right)$ dont le sol, par exception, était recouvert de mortier de tuileau, présentait dans un angle un massif de pierres liées à l'argile $(1 \mathrm{~m} \times 1,50 \mathrm{~m})$ qui servait de base aux jumelles d'un pressoir à levier. Dans l'axe du massif, une fosse quadrangulaire doit correspondre au logement de son contrepoids. La pièce, qui était utilisée à la fois comme fouloir et comme pressoir, était reliée par un tuyau de plomb à un dolium enterré dans le substrat et contenant 350 litres environ (fig. 26). La pièce $n^{\circ} 5$ devait servir de cella vinaria, car on y a découvert les fragments de dix dolia. Dans son angle sud-est se trouvait un grand foyer creusé dans le rocher (longueur : $1,40 \mathrm{~m}$; largeur : $0,60 \mathrm{~m}$ ) : il pourrait avoir été utilisé 


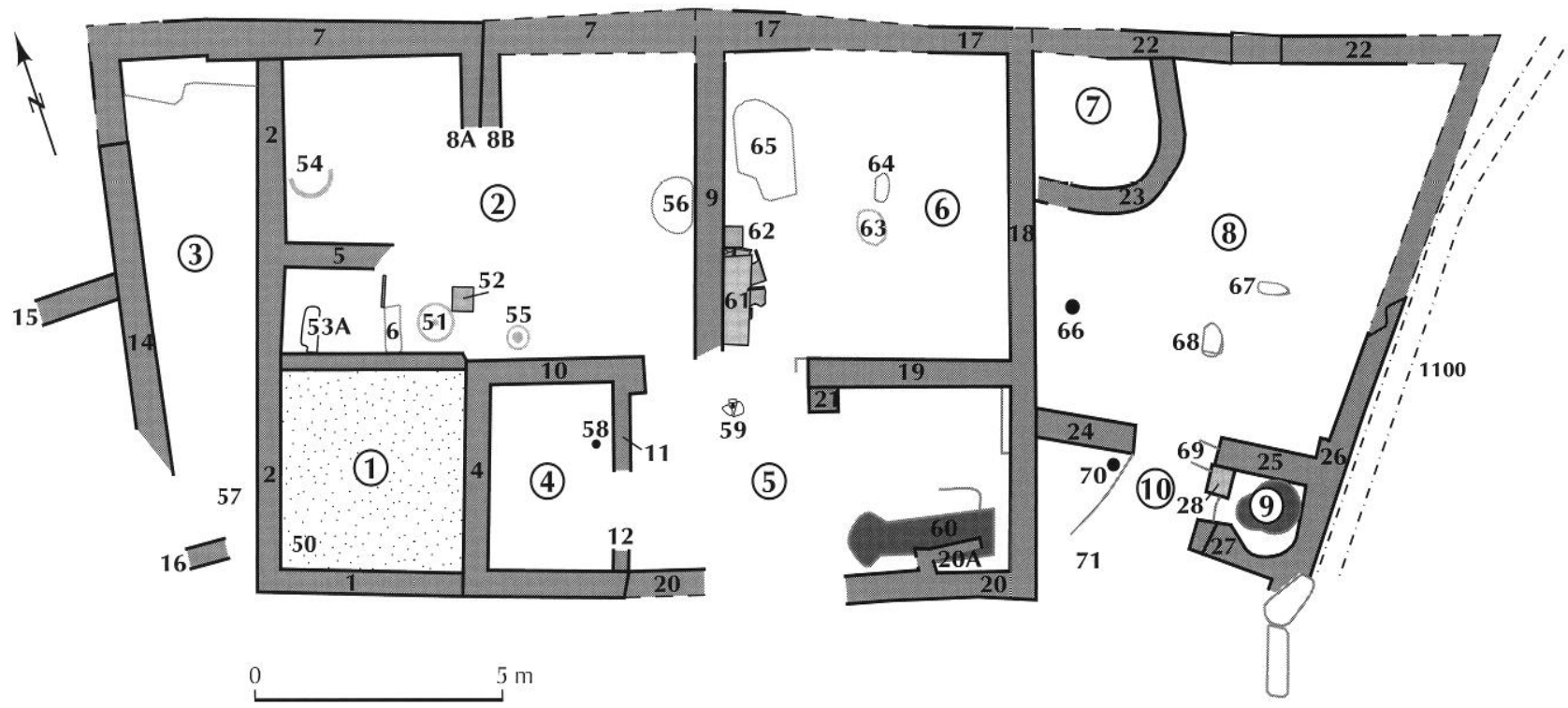

Fig. 25 - Plan de la ferme de Barresse au Muy, Var (plan F. Laurier, Centre archéologique du Var).

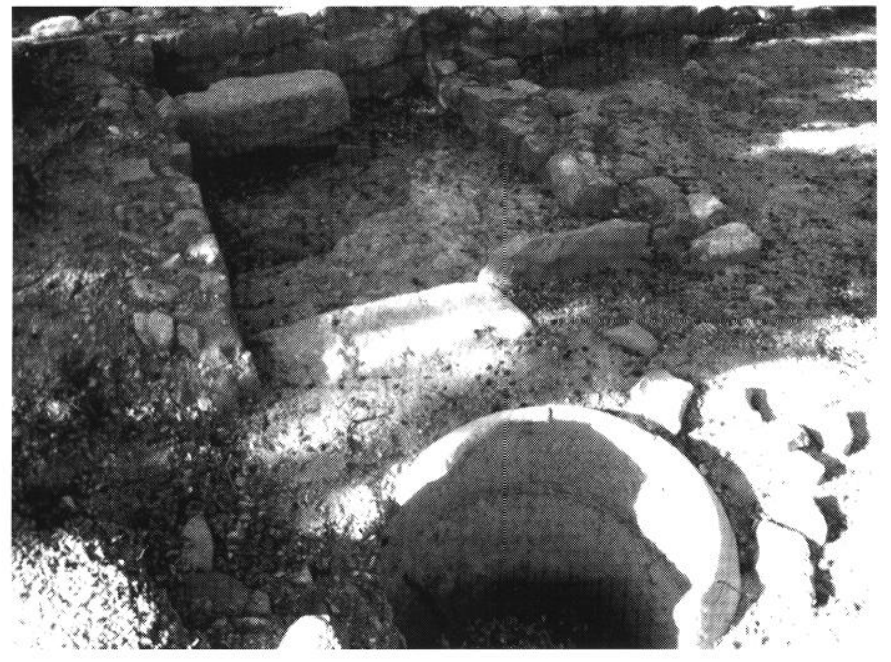

Fig. 26 - Le pressoir à vin de Barresse au Muy, Var (cliché J. Bérato).

pour chauffer un chaudron destiné à la cuisson du defrutum.

Cette ferme modeste faisait probablement partie d'un groupe d'habitations rurales, formant peut-être un véritable village établi dans les collines dominant la plaine.

\section{Forbin à Aix-en-Provence (Bouches-du-Rhône)}

Deux villae situées à la sortie sud-est d'Aquae Sextiae ont été fouillées par N. Nin. La première comporte une installation viticole construite vers le milieu du $\mathrm{I}^{\mathrm{er}} \mathrm{s}$. après J.-C. et abandonnée vers la fin du $\mathrm{II}^{\mathrm{c}} \mathrm{s}$. (un fouloir, un pressoir, deux cuves, nombreux dolia). La villa de Forbin 2 fut occupée du milieu du $\mathrm{I}^{\mathrm{er}}$ s. après J.-C. à la fin du IV $\mathrm{e}$. La fouille a porté sur la pars urbana qui fut transformée en cellier à dolia au milieu du $\mathrm{II}^{\mathrm{e}} \mathrm{s}$. La production de vin semble avoir duré jusqu'au milieu du IV's.

\section{L'Ormeau A à Taradeau (Var)}

Deux fermes voisines appartenant à un hameau ont été fouillées en 1980 par le Centre archéologique du Var (CAV) (Brun, Congès, Pasqualini dir., 1993, p. 105-250). Chacune comportait une huilerie qui avait fonctionné entre la période flavienne et la fin du $\mathrm{II}^{e} \mathrm{~s}$. Mais la ferme $\mathrm{A}$ possédait en outre un fouloir à vin caractérisé par une dalle de béton dotée d'un déversoir en grès. Le jus s'écoulait dans des baquets de bois placés dans une fosse sous le déversoir. Les installations de vinification elles-mêmes, probablement situées sous une route, n'ont pas été dégagées.

\section{Sivier à Istres (Bouches-du-Rhône)}

Le site de Sivier, situé au nord de l'étang de l'Olivier, est connu par des prospections conduites par F. Trément (Gateau et al., 1996) et par une fouille partielle effectuée en 1999-2000 par F. Marty (2001). Il comprenait une habitation, une installation vinicole taillée dans le roc et un 

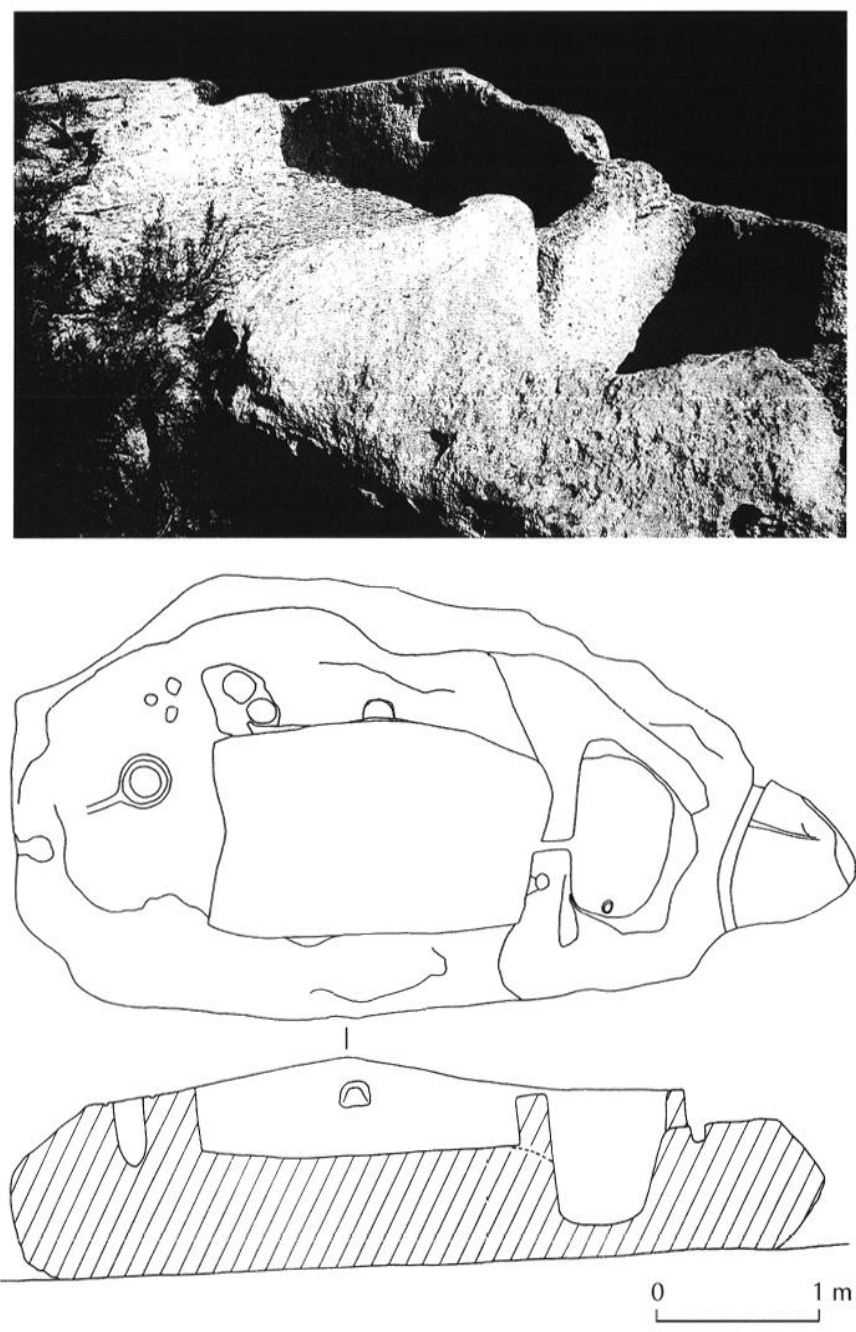

Fig. 27 - L'installation vinicole de Sivier à Istres, Bouches-du-Rhône (d'après Gateau et al., 1996, fig. 83 ; cliché F. Trément).

atelier de potier d'amphores vinaires (fig. 27). Le matériel archéologique trouvé en prospection comporte des céramiques datables du $\mathrm{I}^{\mathrm{er}} \mathrm{s}$. avant J.-C. au $\mathrm{V}^{\mathrm{e}} \mathrm{s}$. après J.-C. Un four quadrangulaire de $3,55 \mathrm{~m}$ de côté a fonctionné durant la seconde moitié du $\mathrm{I}^{\text {er }} \mathrm{s}$. Il a produit des amphores Gauloise 4 et 5 principalement, ainsi que des matériaux de construction. L'installation vinicole rupestre ressemble à celles du Proche-Orient, de Grèce, d'Italie et de Kabylie : un fouloir rectangulaire, mesurant 2,10 m sur $1,25 \mathrm{~m}$ pour une profondeur de $0,30 \mathrm{~m}$ environ, est relié par une saignée à un petit bassin de recueil en forme d'ovale irrégulier mesurant $1 \mathrm{~m}$ dans sa grande largeur et profond de 0,60 m environ (contenance: 250 litres environ). Une niche semi-circulaire, large d'une vingtaine de centimètres, est ménagée dans la paroi nord du fouloir, à $0,30 \mathrm{~m}$ au-dessus du fond. Elle servait à fixer l'extrémité

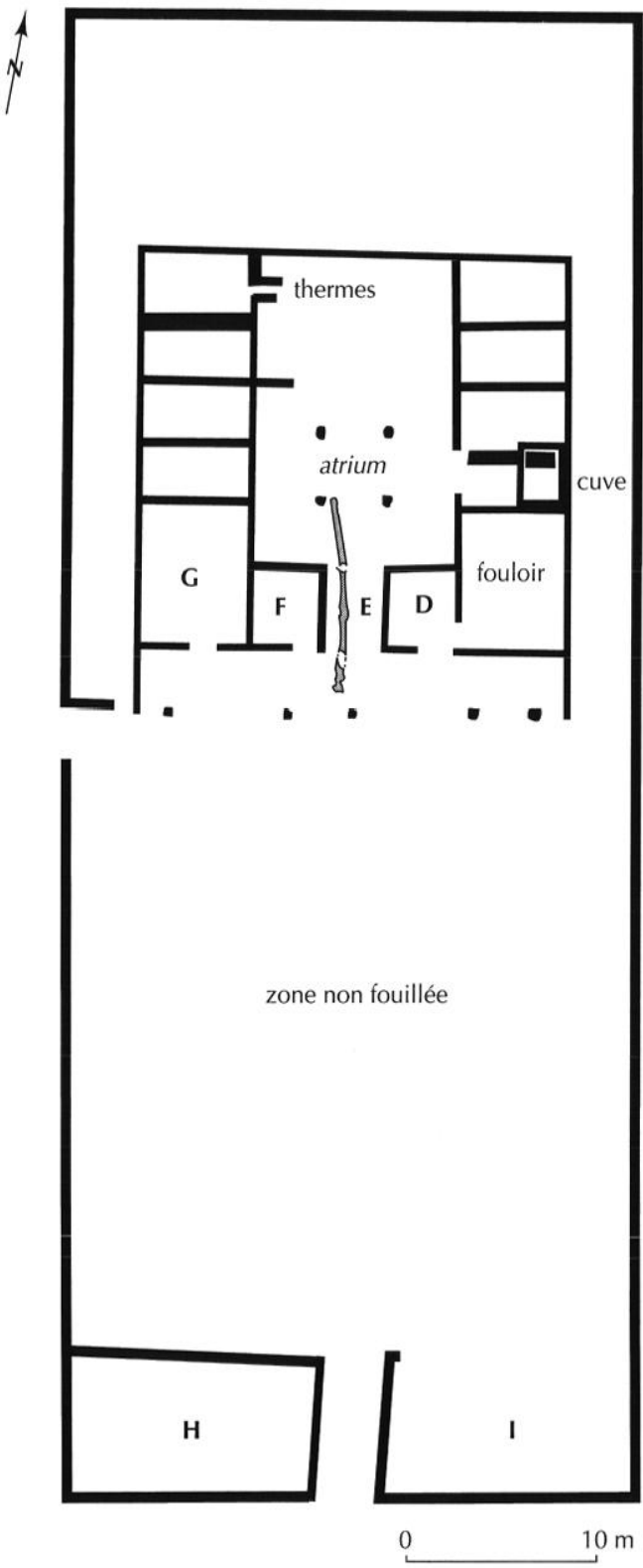

Fig. 28 - Plan de la villa du Suveret à Saint-Raphaël, Var (d'après V. Lelièrure, 1993).

d'une poutre que l'on utilisait comme levier pour presser le marc après l'avoir foulé. L'ensemble devait être protégé par une couverture légère: des trous de poteaux sont creusés à la périphérie du fouloir.

\section{Le Suveret à Saint-Raphaël (Var)}

Le site a été dégagé par V. Lelièvre à l'occasion d'une fouille d'urgence en 1988 (Lelièvre, 1993). Un enclos mesurant $70 \mathrm{~m}$ de long sur $30 \mathrm{~m}$ de large entourait 
un bâtiment central qui a connu deux états. Lors de l'état 1 , édifié au début du $\mathrm{I}^{\mathrm{er}} \mathrm{s}$. de notre ère, la villa formait un carré de $22 \mathrm{~m}$ de côté comportant une galerie en façade. Le centre du bâtiment était occupé par un atrium tétrastyle, l'aile ouest était consacrée à l'habitation et aux bains, et l'aile est abritait l'installation vinicole: fouloir à vin $(6,25 \mathrm{~m} \mathrm{x} \mathrm{4,80} \mathrm{m})$ relié par un tuyau de plomb à une cuve mesurant $2,40 \mathrm{~m}$ de longueur, $1,70 \mathrm{~m}$ de largeur et $1,20 \mathrm{~m}$ de profondeur (contenance: 4000 litres environ) (fig. 28). Mais on n'a retrouvé aucun vestige permettant de déceler une cella vinaria (pas de dolia defossa). À la suite d'un incendie qui eut lieu dans le courant du ${ }^{\text {er }}$ s., le plan fut modifié : plusieurs pièces furent bâties dans l'atrium primitif qui perdit sa fonction, de même que le fouloir et la cuve à vin dans laquelle on construisit un mur. La date haute de la construction et le plan de la villa nettement inspiré de modèles italiques semblent indiquer qu'il s'agit d'un domaine édifié par un colon italien de Forum Iulii, tout proche. L'abandon du site semble être intervenu avant la fin du $I^{\text {er }} s$. après J.-C.

\section{Tout-Égau à Taradeau (Var)}

Une fouille de sauvetage effectuée par J. Bérato (CAV) en 1991-1992 a dégagé une petite ferme implantée sur un coteau (Brun, Congès, Pasqualini dir., 1993, p. 251-266). L'établissement a connu trois états d'occupation entre le milieu du ${ }^{\text {er }} \mathrm{s}$. avant J.-C. et la fin du I ${ }^{\mathrm{er}} \mathrm{s}$. après J.-C. Lors du troisième état (seconde moitié $\mathrm{du} \mathrm{I}{ }^{\mathrm{er}} \mathrm{s}$.), on construisit une petite installation vinicole dont subsiste sculement le fond d'une cuve bétonnée.

\section{$2^{\mathrm{e}}$ GROUPE : INSTALLATIONS COMPLEXES}

Une seconde classe d'installations vinicoles regroupe celles qui comportent deux à quatre pressoirs et un grand nombre de dolia.

\section{Le Grand Loou I à La Roquebrussanne (Var)}

La villa du Grand Loou I a été intégralement fouillée par (. Congès et J.-P. Brun entre 1981 et 1983 (Brun, 1999 , p. 620-626). Ses origines remontent au milieu du $\mathrm{I}^{\mathrm{er}} \mathrm{s}$. avant $\mathrm{J}$.-C., mais c'est seulement à partir du début du II $^{\mathrm{e}} \mathrm{s}$. que l'on trouve des vestiges liés à la production de vin. Les installations les plus remarquables ont été

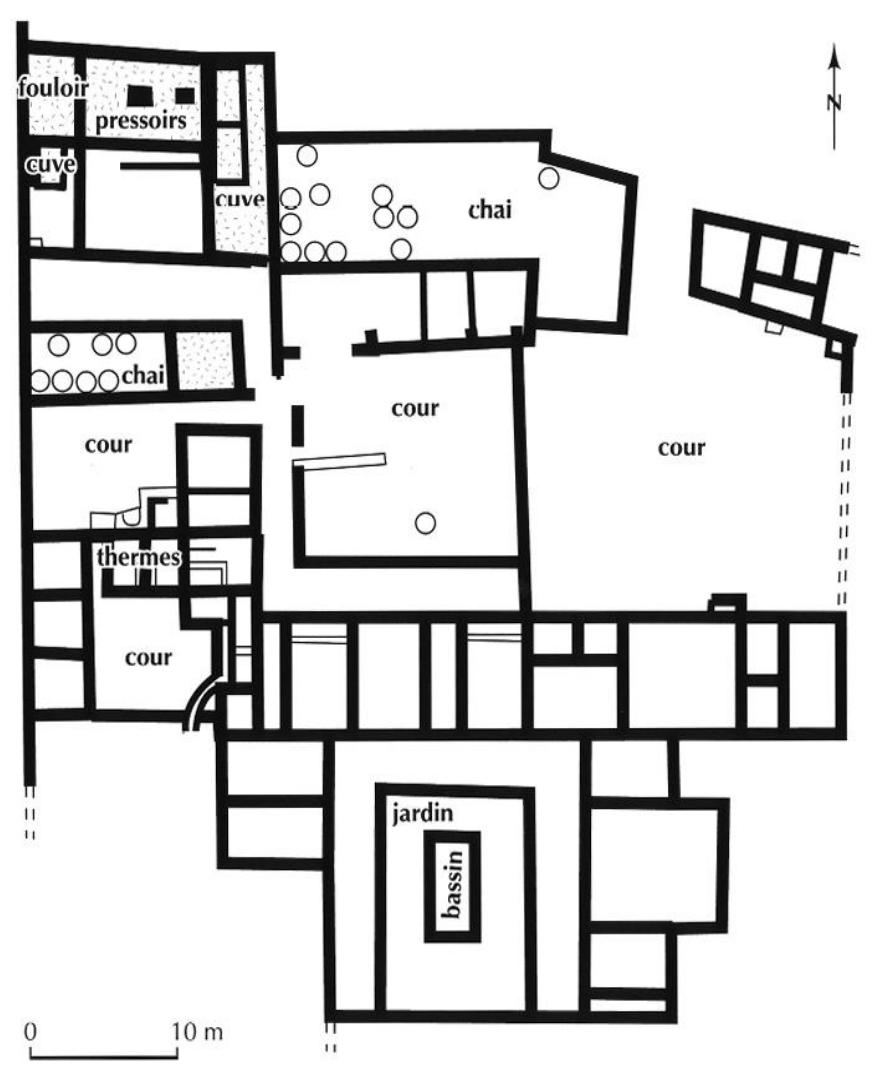

Fig. 29 - Plan de l'installation vinicole de la villa du Crrand Loou à La Roquebrussanne, Var (plan J.-P. Brun et (G. Congès).

édifiées vers le milieu du siècle. Elles comprenaient un fouloir au sol bétonné et son bassin de recueil, deux pressoirs à levier et contrepoids et leurs bassins, et un chai susceptible de loger 10 dolia defossa auxquels s'ajoutaient d'autres jarres antérieurement mises en place (fig. 29). Dans la seconde moitié du II ${ }^{e}$ s., on bâtit un nouveau chai abritant une cinquantaine de dolia. Les capacités de vinification se seraient donc élevées à $1100 \mathrm{hl}$ environ. La villa, peut-être absorbée par un domaine voisin, fut abandonnée vers la fin du II's s.

\section{Les Mesclans à La Crau (Var)}

La pars fructuaria d'une villa très détériorée par les labours a été totalement dégagée en 1996 par le CAV (Brun, Borréani, 1998) (fig. 30 ct 31). Les bâtiments ont été édifiés dans la première moitié du II ${ }^{e}$ s. après J.-C. et occupés jusqu'au troisième quart du III $^{e} \mathrm{~s}$. Autour d'une cour, se trouvaient, au nord-est, des pièces d'habitation, au nord-ouest, unc installation vinicole, à l'ouest, une huilerie et, au sud, un moulin hydraulique. L'installation 


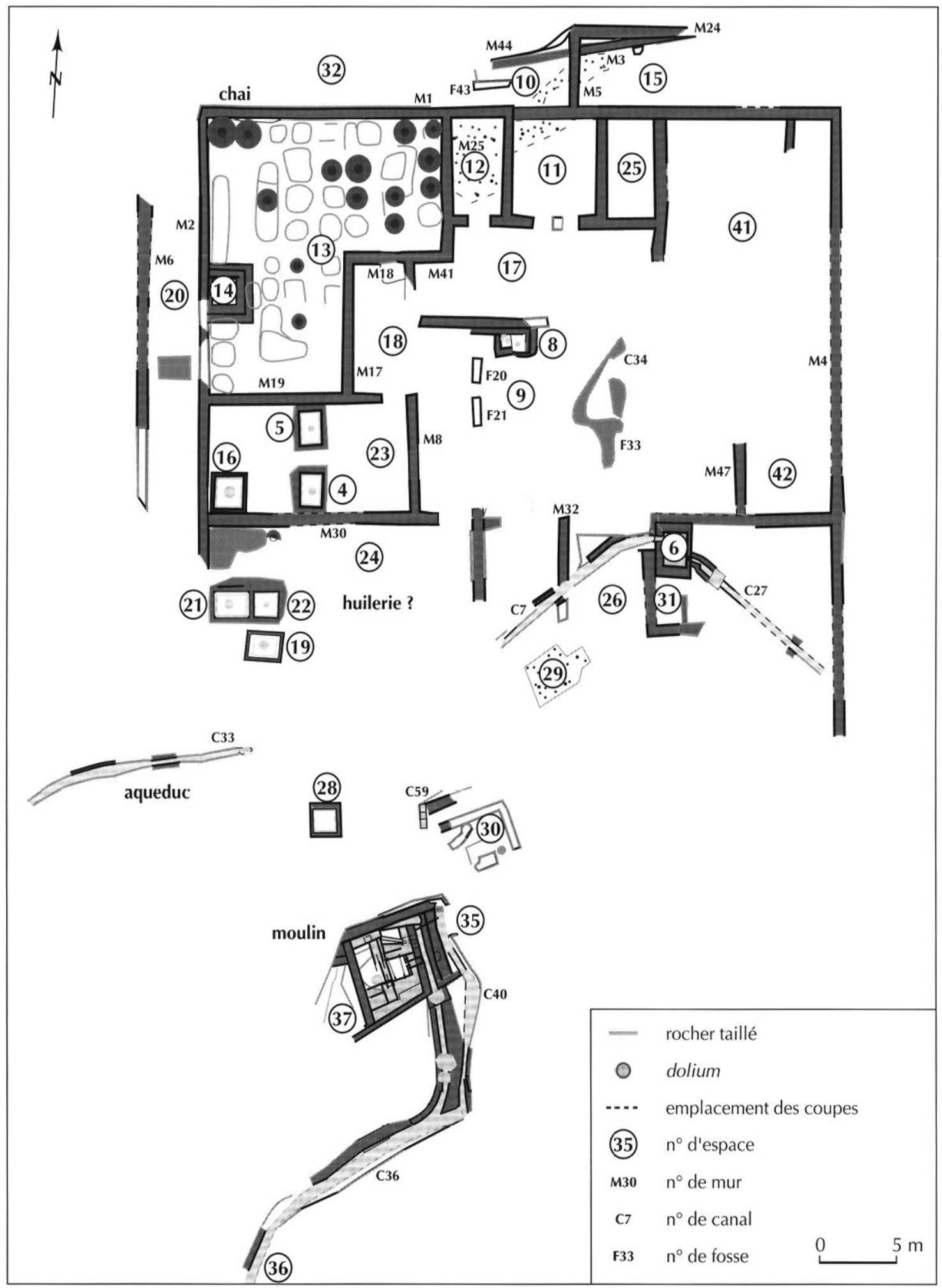

Fig. 30 - Plan de la villa des Mesclans à La Crau, Var (plan F. Laurier, Centre archéologique du Var).

vinicole occupait deux salles: au nord, un entrepôt $\left(n^{\circ} 13\right)$ où étaient enterrés une cinquantaine de dolia et, au sud, la salle des fouloirs et des pressoirs $\left(n^{\circ} 23\right)$ qui comptait trois cuves bétonnées comportant des cuvettes 


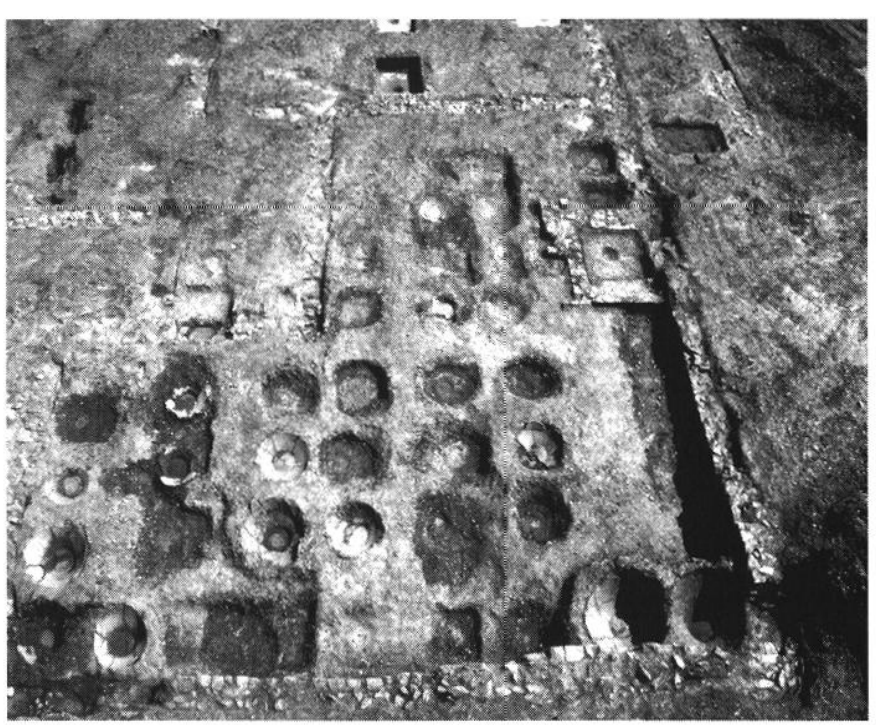

Fig. 31 - Linstallation vinicole de la villa des Mesclans à La Crau, Var (cliché J.-P. Brun). de vidange $\left(\mathrm{n}^{\circ \mathrm{s}} 4,5,16\right)$. Deux dolia étaient suffisamment bien conservés pour estimer leur contenance autour de $19 \mathrm{hl}$. Si toutes les jarres étaient similaires, le chai possédait une capacité maximale de vinification de $950 \mathrm{hl}$. La villa des Mesclans est située à $4 \mathrm{~km}$ du Collet Long où se trouve un atelier d'amphores Gauloise 4 qui servaient peut-être à expédier le vin du domaine.

\section{Pardigon 3 à Cavalaire-sur-Mer (Var)}

Au fond de la baie de Cavalaire, en arrière de la plage, une villa viticole fit l'objet d'une fouille d'urgence en 1985-1986 par le CAV (Brun, 1999, p. 324-330) La villa fut occupée entre le dernier quart du $\mathrm{I}^{\mathrm{cr}} \mathrm{s}$. et le début du III $\mathrm{c}$. après J.-C. Les bâtiments, couvrant une superficie de $2000 \mathrm{~m}^{2}$, sont centrés sur un jardin entouré d'un péristyle et comportent à l'est une aile thermale et au

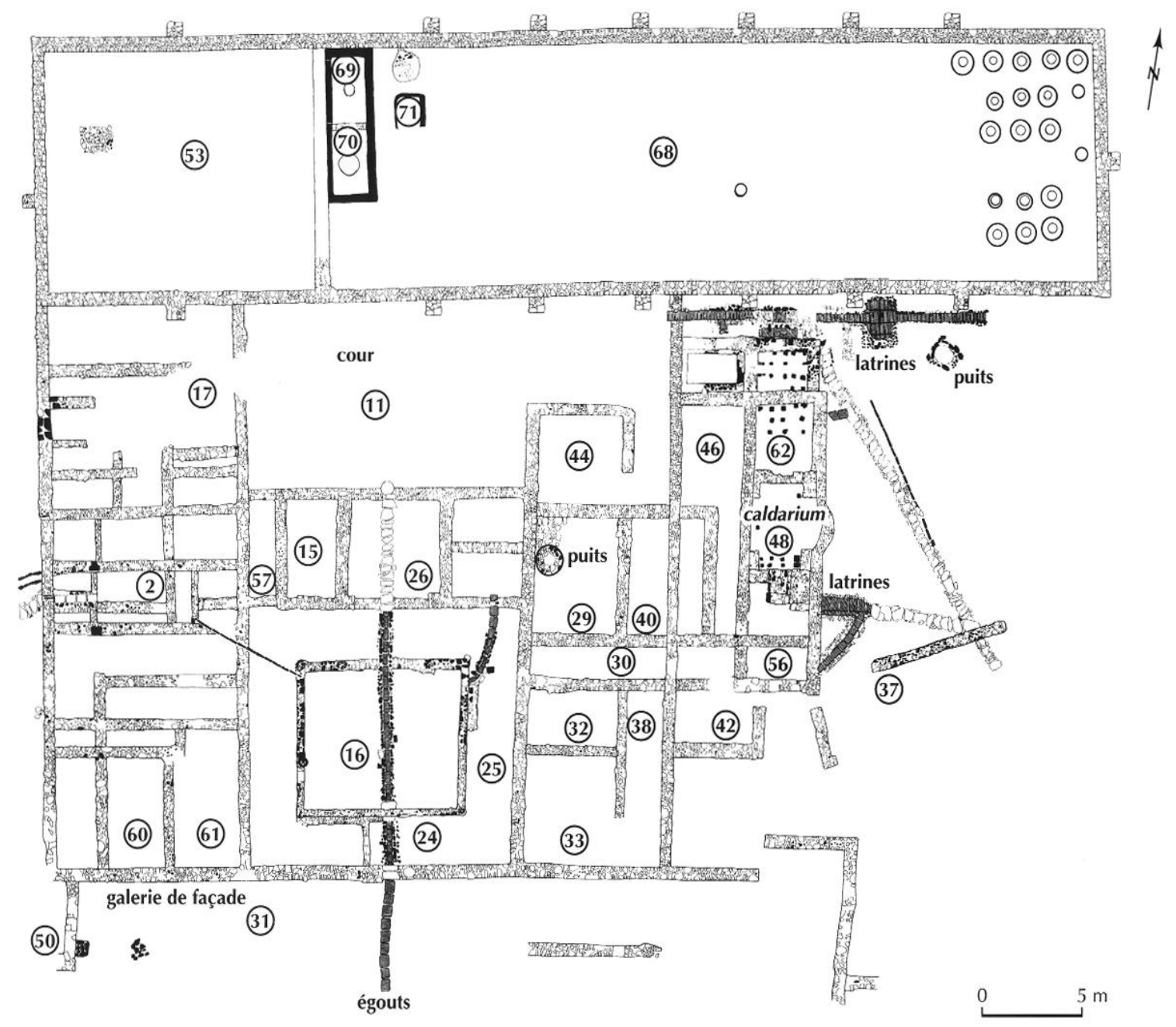

Fig. 32 - Plan de la villa de Pardigon 3 à Cavalaire-sur-Mer, Var (plan J.-P. Brun). 


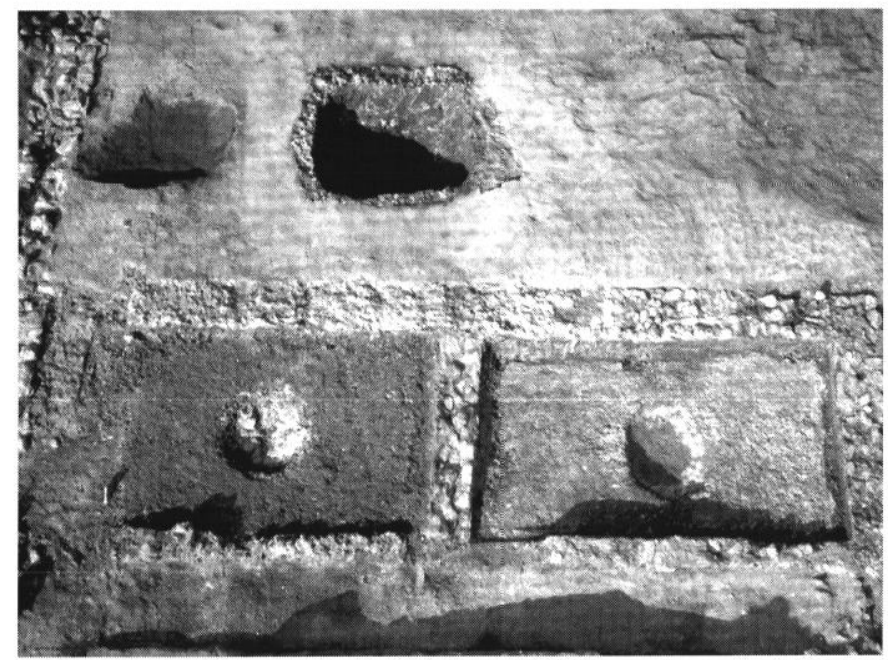

Fig. 33 - Les cuves et le defrutarium de la villa de Purdigon 3 à Cavalaire-sur-Mer, Var (cliché J.-P. Brun).

nord un vaste chai (fig. 32). Le chai, un bâtiment à contreforts long de $52 \mathrm{~m}$ et large de $12 \mathrm{~m}$, abritait à l'ouest les fouloirs et le pressoir, et à l'est les cuves et les dolia. Les sols des presses n'étaient pas conservés, mais deux cuves jumelles, dont l'étanchéité avait été refaite trois fois, étaient accolées au mur des chambres de presse. À côté, une petite cuve voisinait avec la base d'une chaudière : il semble qu'il s'agisse d'unc installation dc réduction du moût, un defrutarium (fig. 33). Dans la partie orientale, mieux préservée par l'angle des murs, vingt fonds de dolia subsistaient; leur répartition régulière permet une estimation de leur nombre primitif : 115 environ, soit une capacité de vinification de l'ordre de 1100 à $2000 \mathrm{hl}$. La villa semble avoir été abandonnée dans la première moitié du $\mathrm{III}^{\mathrm{e}} \mathrm{s}$. après J.-C. et ses bâtiments ont dû servir de carrières de pierre pour certaines constructions dans la villa voisine de Pardigon 2 (La Croix-Valmer).

\section{Rue du Port à Cavalaire-sur-Mer (Var)}

À l'extrémité ouest de la baie de Cavalaire, une autre villa viticole possédait des installations encore plus importantes (Brun, Oberti, 1993; Brun, 1999, p. 330-332). Fouillée en 1993, lors de la réfection de la rue du Port par le CAV, elle comptait quatre fouloirs et pressoirs à vin, trois cuves et un cellier rempli de dolia defossa. Les quatre pressoirs comportaient des sols en béton de tuileau pourvus de blocs d'assise des

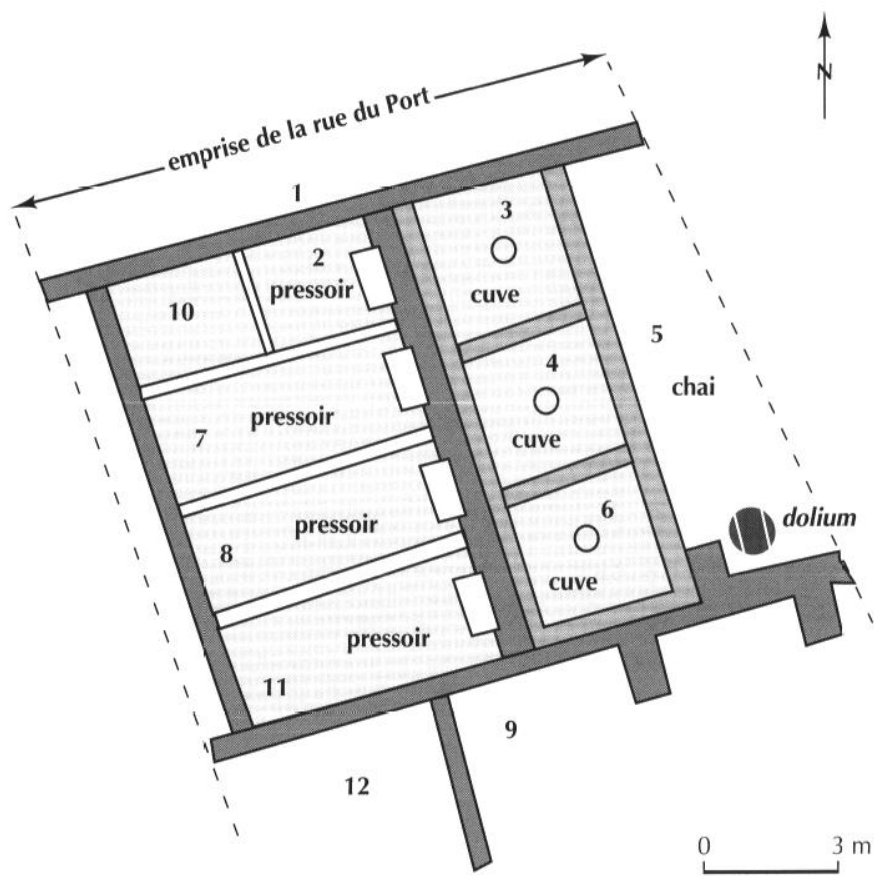

Fig. 34 - Plan du chai de la rue du Port à Cavalaire-sur-Mer; Var (plan J.-P. Brun).

jumelles (fig. 34). Les cuves, placées en tête des pressoirs, mesuraient environ $2,80 \mathrm{~m} \times 2,80 \mathrm{~m}$ pour une profondeur moyenne de $0,50 \mathrm{~m}$, soit une capacité maximalc de 4000 litres chacune. À l'est s'étendait le cellier $\mathrm{n}^{\circ} 5$, bordé au sud et très certainement au nord par un mur à puissants contreforts destinés à supporter les poussées de la couverture. Il abritait un nombre indéterminé de dolia. Un seul a pu être découvert en place, toute la partie nord-est du chai ayant été détruite par un immeuble voisin. La construction du chai doit être placée vers la fin du I ${ }^{\text {er }}$ s. ou au début du II $^{\text {e }}$ s. après J.-C. ; l'abandon de la production vinicole, l'enlèvement d'une partie des dolia et le comblement partiel des cuves par des déchets sont clairement attribuables au deuxième quart du IV $\mathrm{s}$.

\section{Saint-Martin à Taradeau (Var)}

La villa de Saint-Martin fait l'objet d'une fouille programmée du CAV dirigée par J. Bérato depuis 1995 (Brun, 1999, p. 766-771).

Le domaine fondé dans la seconde moitié du $I^{\text {er }} s$. avant J.-C. a peut-être appartenu successivement à un certain P. Varius (sous Auguste), puis à des Valerii originaires de Gênes (dans la première moitié du $\mathrm{I}^{\mathrm{er}} \mathrm{s}$.). La 


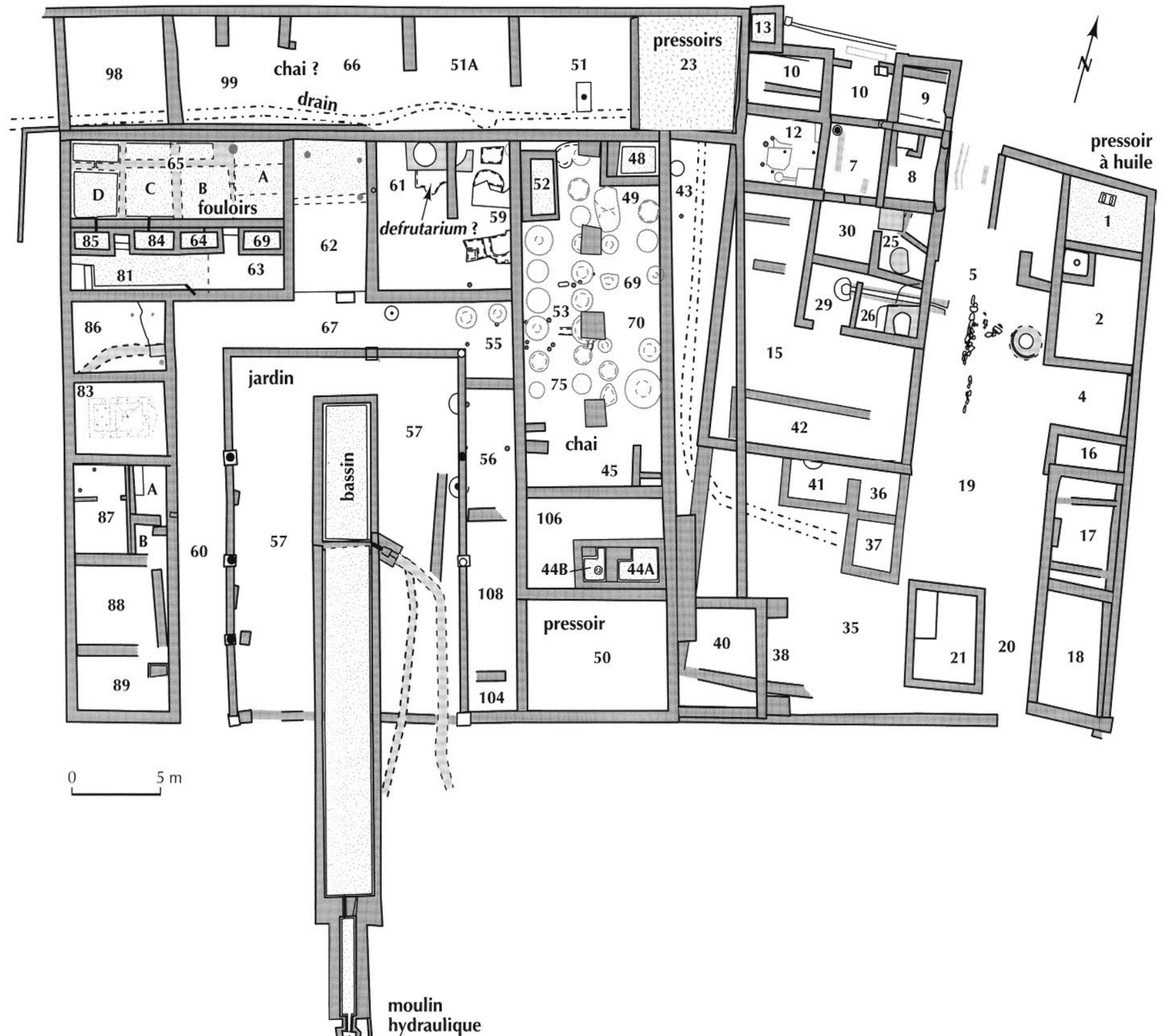

Fig. 35 - Plan de la villa de Saint-Martin à Taradeau, Var (plan F. Laurier, Centre archéologique du Var).

villa connut un développement progressif et, à la fin du $\mathrm{I}^{\mathrm{er}} \mathrm{s}$. après J.-C., présentait un plan classique : ailes résidentielles entourant un jardin bordé d'un portique et orné d'un bassin. Dans le courant du $\mathrm{II}^{\mathrm{c}} \mathrm{s}$., probablement après le milieu du siècle, la pars urbana fut désaffectée et transformée en installation vinicole (fig. 35). Sur les anciennes pièces d'habitation, on édifia des fouloirs, des pressoirs et des cuves à vin, et le bassin d'agrément fut converti en moulin hydraulique. Ce réaménagement, qui semble le fait d'un nouveau propriétaire, consista à édifier quatre fouloirs et quatre cuves ( $\mathrm{n}^{\mathrm{os}} 65$ et 81 ) fonctionnant par paires (fig. 36). Chaque ensemble comportc, au nord, dcux aires en mortier avec boudin d'étanchéité et, au sud, une cuve. Les quatre cuves mesurent $0,85 \mathrm{~m}$ $\mathrm{x} 1,90 \mathrm{~m}$ et $0,85 \mathrm{~m}$ de profondeur. La contenance de chacune d'elles est de $13 \mathrm{hl}$ environ. À l'intérieur de la pièce 65 , des murets délimitent les fouloirs auxquels on accédait par des escaliers à deux marches situés entre les cuves 64 et 69 et entre les cuves 84 et 85 . Plus à l'est, la pièce 23 fut remblayée et transformée en pressoir à deux 


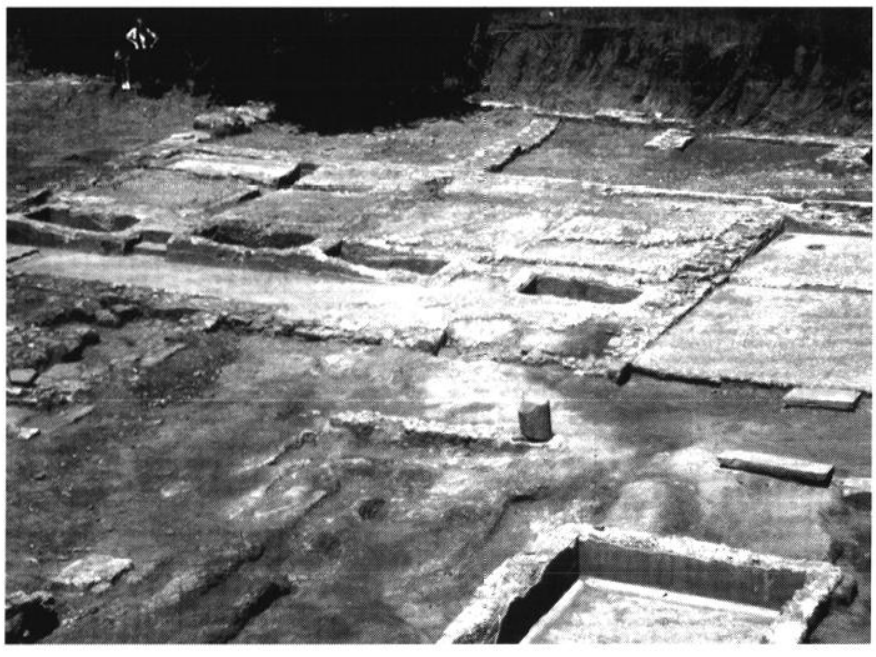

Fig. 36 - Fouloirs et curves de la villa de Saint-Martin à Taradeau, Var (cliché J. Bérato, Centre archéologique du Var).

appareils. Dans une première phase, ils ont dû posséder des machineries à treuil qui furent transformées ultérieurement par substitution d'une vis. Ces pressoirs étaient reliés à des cuves (52 et 48 ) situées dans le chai $45 / 53$. La cuve 48 mesure $1,80 \mathrm{~m} \times 2,10 \mathrm{~m}$ et sa contenance minimale est de 2700 litres. Elle comporte un escalier et une cuvette de vidange. La cuve 52 mesure $1,40 \mathrm{~m} \times 3 \mathrm{~m}$ et sa contenance est de 3800 litres environ. Cette cuve a été l'objet d'une réfection durant son utilisation, comme en témoigne le piquetage du premier enduit. Le chai,

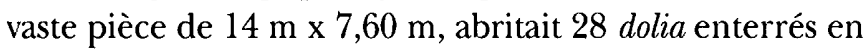
deux rangées centrales. Si l'on donne à chacun une contenance de l'ordre de $15 \mathrm{hl}$, la capacité de vinification atteindrait les $420 \mathrm{hl}$. Deux murets parallèles s'appuient contre le mur ouest du chai; leur disposition pourrait évoquer un support de foudre.

L'installation viticole était complétée par un defrutarium construit dans la pièce 61 . Il s'agit d'une structure rectangulaire comportant deux bases de foyers circulaires et qui devait supporter des chaudrons de cuisson du moût.

Une autre unité viticole, plus petite, semble avoir été construite peu de temps après la première dans l'angle sud-est de l'ancienne pars urbana. Elle comporte probablement un fouloir et un pressoir (pièces 50 et 40 ) et deux cuves contiguës $44 \mathrm{~A}$ et $44 \mathrm{~B}$, qui mesurent $1,80 \mathrm{~m}$ x 2,40 m (cuve 44A) et $1,40 \mathrm{~m} \times 1,80 \mathrm{~m}$ (cuve 44B). La profondeur conservée étant de $0,90 \mathrm{~m}$, leur contenance s'élevait à 3900 et 2300 litres au moins.
La production de vin semble avoir été continue durant les $\mathrm{II}^{\mathrm{e}}$, III ${ }^{\mathrm{e}}$ et $\mathrm{IV}^{\mathrm{e}} \mathrm{s}$. Ce n'est qu'au début du V`es. que les cuves et les dolia furent comblés. Mais la villa continua cependant d'être occupée jusqu'au VI's. après J.-C.

\section{Les Toulons à Rians (Var)}

La villa des Toulons, anciennement connue, a fait l'objet d'une fouille programmée entre 1993 et 1996 menée par le CAV (Brun, Congès, 1994 ; Brun, 1999, p. 597-604). Les bâtiments de la villa couvrant environ 1 ha sont implantés sur une éminence. Au sud-ouest, en bordure du plateau, on trouve les vestiges de la pars urbana recouverts par une ferme et, au nord-est, ceux de la pars fructuaria qui couvre une superficie de $8000 \mathrm{~m}^{2}$ (fig. 37). Les bâtiments agricoles, construits dans le dernier quart du Ir ${ }^{\text {er }}$. après J.-C., s'organisaient autour de deux cours. Dans la cour nord ouvraient deux bâtiments symétriques abritant chacun deux fouloirs et deux pressoirs à levier actionnés par treuils, fixés sur d'énormes contrepoids. Le moût s'écoulait par des tuyaux de plomb dans des cuves bétonnées situées en contrebas du côté sud. Chaque ensemble était doté de quatre cuves susceptibles de contenir entre 4700 et 7900 litres environ.

La cour sud était bordée sur trois côtés par de grands celliers à dolia. Large de $9 \mathrm{~m}$, l'aile nord avait une longueur de 51,90 m (fig. 38). Les ailes est et ouest, en grande partie détruites, mesuraient au moins $40 \mathrm{~m}$ de long et peut-être autant que l'aile nord. Les jarres étaient disposées en deux séries de deux rangées parallèles, séparées par un espace de circulation. Les estimations effectuées à partir des zones bien conservées montrent que le chai devait abriter une quantité de dolia supérieure à 200. Comme la contenance moyenne de chacun est de l'ordre de $15 \mathrm{hl}$, la capacité totale de stockage devait dépasser les $3000 \mathrm{hl}$. Au début du $\mathrm{III}^{e}$ s., les pressoirs à câble furent transformés en pressoirs à vis. Les anciens contrepoids furent adaptés et de nouveaux, cylindriques, furent taillés. Dans le courant du III ${ }^{\mathrm{e}}$ s., la production de vin diminua fortement. Des dolia furent retirés, probablement pour être vendus. Le pressoir oriental et toute la partie orientale du chai furent désormais laissés à l'abandon et, au début du IV $\mathrm{V}^{\mathrm{e}}$ s., la partie encore active du chai fut transformée en pièce d'habitation. Vers la fin $d u I^{\mathrm{c}}$ s. ou au début du $V^{\mathrm{c}} s$., 


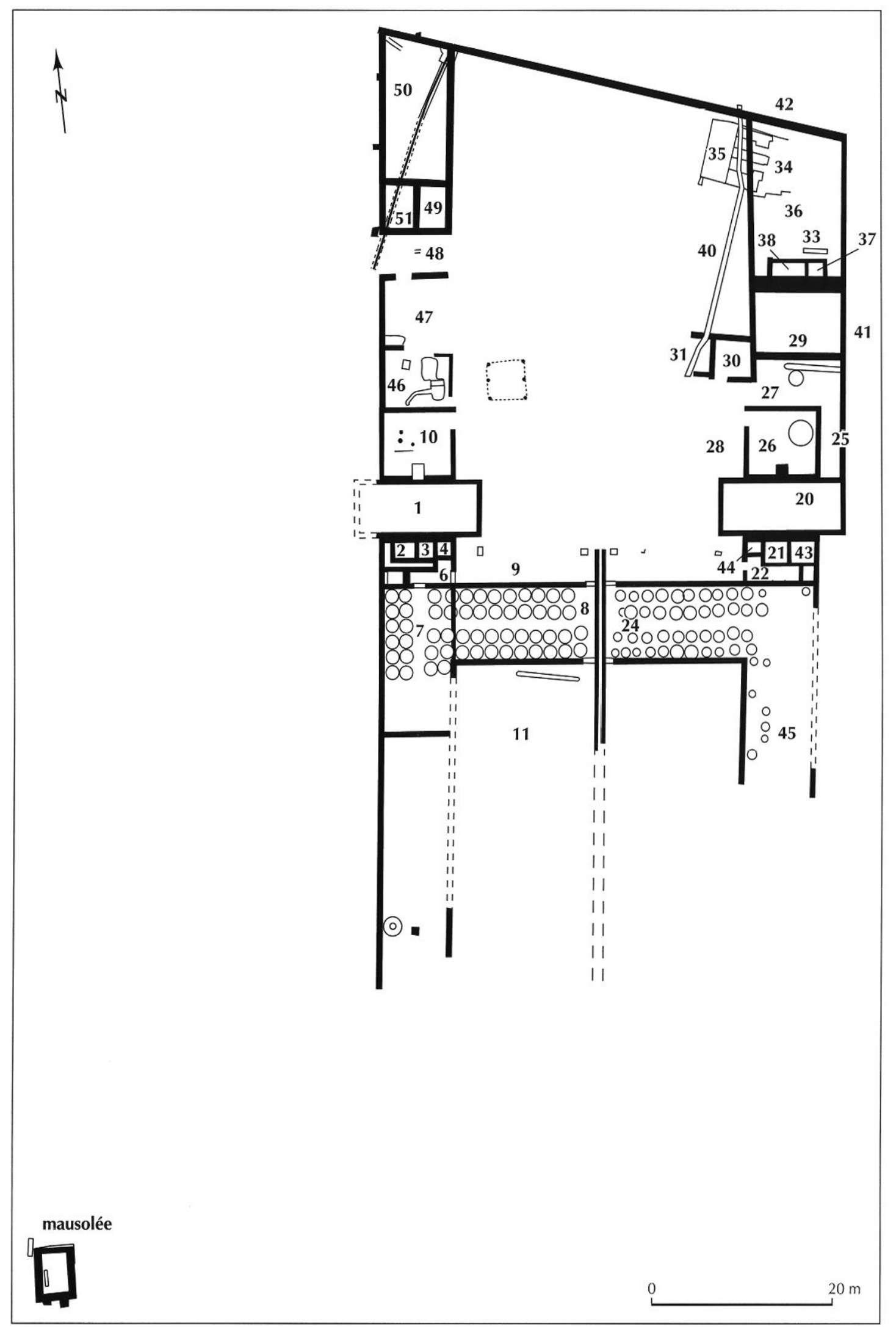

Fig. 37 - Plan de l'installation vinicole de la villa des Toulons à Rians, Var (plan F. Laurier, Centre archéologique du Var). 


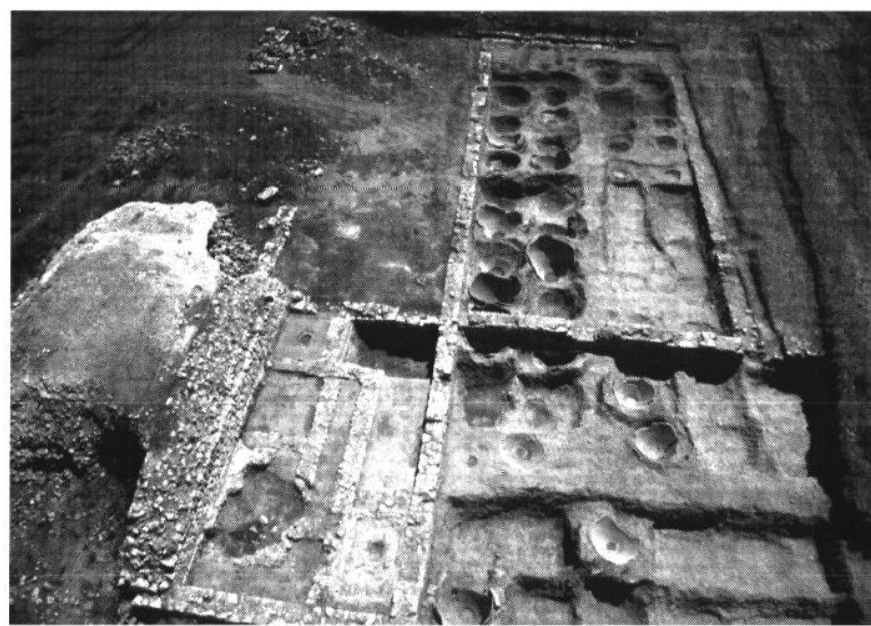

Fig. 38 - L'aile nord du chai de la villa des Toulons à Rians, Var (cliché J.-P. Brun).

tous les pressoirs furent détruits. Les cuves furent arasées et les appareils démantelés pour récupérer le bois et les métaux, notamment le fer et le plomb.

\section{Tourville à Saignon (Vaucluse)}

En 1998, des travaux de terrassements ont partiellement détruit une villa comportant une pars urbana (non dégagée), une huilerie et un chai à vin (Carru, Kauffmann, 1999). Ce dernier était équipé de deux fouloirs se déversant dans des dolia enterrés et d'un grand cellier. Le bâtiment, long de 23,50 m et large de $8 \mathrm{~m}$, abritait une cinquantaine de dolia disposés en deux rangées. La villa semble avoir été occupée surtout au $\mathrm{II}^{\mathrm{e}} \mathbf{s}$. et dans la première moitié du III $^{\mathrm{e}} \mathrm{s}$. Elle a été incendiée dans la seconde moitié du III ${ }^{\mathrm{e}}$ s., peut-être lors de l'invasion des Alamans.

\section{LES PROCÉDÉS DE VINIFICATION ET LES CAPACITÉS DE PRODUCTION}

Les processus de vinification peuvent être partiellement restitués à partir des vestiges archéologiques. La première opération consistait à fouler le raisin dans une cuve au sol bétonné pourvu d'un exutoire en pierre ou en plomb. Cet équipement est présent dans presque toutes les installations des plus petites (Barresse, L'Ormeau), aux plus grandes (Le Grand Loou, Les Toulons, Saint-Martin). Dans un cas (Sivier), le fouloir est creusé dans la roche. Les fouloirs peuvent faire partie du même ensemble que les pressoirs et partager une même dalle de béton surélevée (Les Toulons) ou bien être établis dans un local séparé. À Saint-Martin, quatre fouloirs sont disposés en batterie à une quinzaine de mètres des deux pressoirs.

Le jus de raisin s'écoulait généralement dans une cuve enduite de béton de tuileau comportant toujours une cuvette de vidange et parfois un escalier (Berthoire à Pignans, Le Vigneret au Castellet). À L'Ormeau toutefois, le moût était recueilli dans des baquets en bois, à Barresse et à Tourville dans un dolium et à Sivier dans une cuve taillée dans le roc. Les rafles étaient ensuite rassemblées et portées sous le pressoir. Quelques petites installations (L'Ormeau, Le Suveret, Tout-Égau) semblent n'avoir pas comporté de pressoir, ou plutôt devait disposer de pressoirs primitifs qui ne laissent pas de vestiges (planches chargées de pierres, sac de toile que l'on tordait). Mais, dès que la villa avait quelque importance, elle était dotée de pressoirs à levier. Durant les deux premiers siècles de l'Empire, deux types de pressoirs furent majoritairement utilisés. Le premier fonctionnait selon les principes décrits par Caton (De l'agriculture, 18-19) : un gros tronc d'arbre horizontal servant de levier est maintenu entre deux forts montants de bois d'un côté et manœuvré à l'autre extrémité à l'aide de câbles tirés par un treuil fixé entre deux autres montants de bois. Ces pressoirs, qui nécessitaient des fondations caractéristiques, sont attestés à Roussivau (Saint-Raphaël) et à Saint-Aygulf (Fréjus). Mais, au I ${ }^{\mathrm{er}} \mathbf{s}$. après J.-C., ce type de pressoir de conception ancienne était concurrencé par les appareils à levier et contrepoids, plus sûrs et performants. Le treuil, au lieu d'être fixé entre deux montants faisant partie de l'édifice, était assujetti à un énorme bloc parallélépipédique en pierre. En actionnant le treuil, on suspendait le contrepoids à l'extrémité du levier et on attendait que la pression exercée par son poids multiplié par le bras de levier agisse sur le marc. C'est le type de pressoir le plus fréquent à partir de la fin du $\mathrm{I}^{\mathrm{er}} \mathrm{s}$., notamment dans les grands domaines comme Les Toulons, Saint-Martin et Le Grand Loou. Les pressoirs à levier et contrepoids étaient aisés à transformer lorsqu'on voulait remplacer le treuil par une vis. L'usage de la vis dans les pressoirs est connu en Italie dès la seconde moitié du I ${ }^{\text {er }} \mathbf{s}$. avant J.-C., mais il ne s'est répandu en Narbonnaise qu'au cours du II $^{e} \mathbf{s}$. après J.-C. et surtout au III $s$. Les grands domaines transformèrent alors leurs 


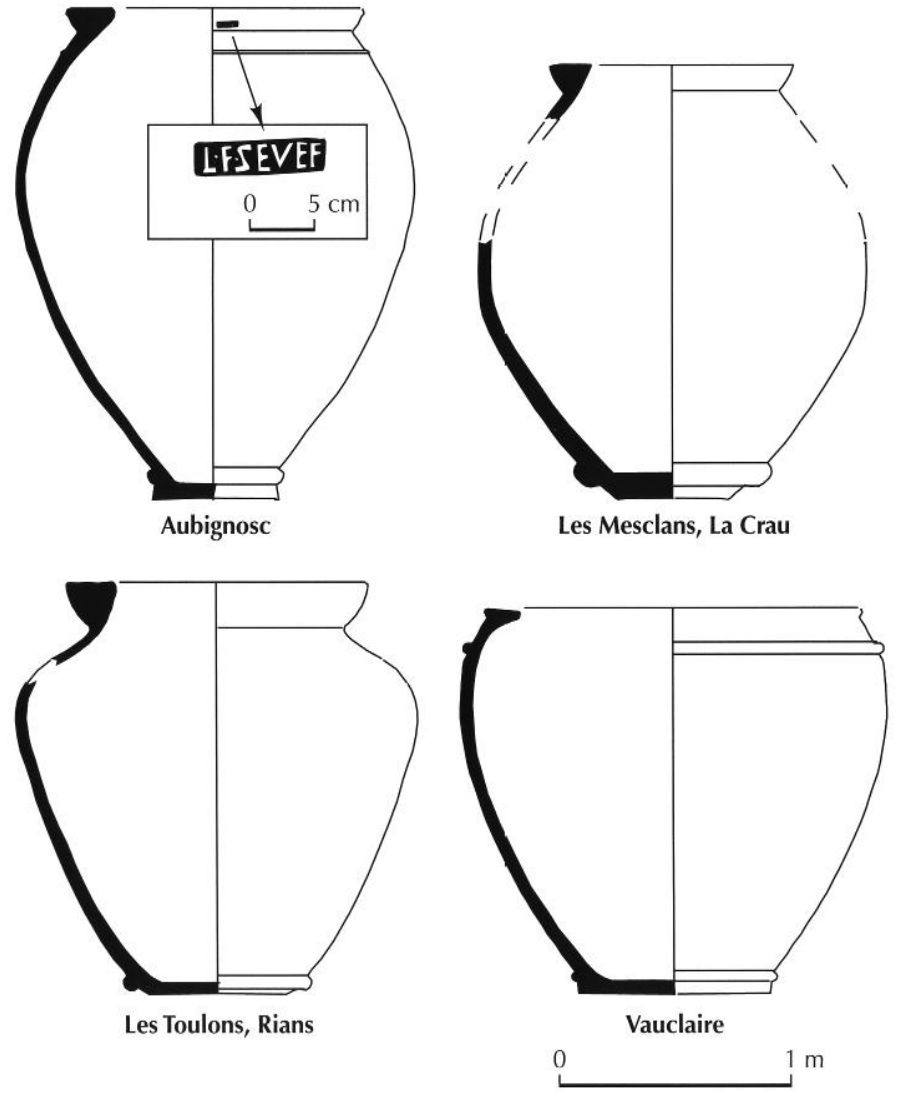

Fig. 39 - Dolia provenant de quatre villae provençales. La marque d'Aubignosc peul être développée en $\mathrm{L}$ (ucius) $\mathrm{F}$ () Seve(rus) f(ecit) (dessin J.P. Brun).

installations (Les Toulons, Saint-Martin et l'installation vinicole du quartier Régine au Puy-Sainte-Réparade, Bouches-du-Rhône, en cours de fouille par P. Chapon, cf. Bilan scientifique de PACA, 2000, p. 132-133) et nombre d'exploitations moins importantes les imitèrent (Le Théron au Cannet-des-Maures, Kierbon à Trets). Dans certains cas, les contrepoids furent remplacés par des blocs aux angles arrondis ou cylindriques qu'il était plus aisé de contourner en poussant les barres de la vis (Les Toulons, Agay à Saint-Raphaël, La Bouverie à Roquebrune-sur-Argens). On n'a pas jusqu'ici retrouvé de vestige de pressoir à vis directe, mais il faut des conditions particulièrement favorables pour que leur empreinte soit conservée (voir infra, p. 137-138).

Le jus de presse s'écoulait dans des cuves analogues à celles placées en bout des fouloirs, à raison d'une cuve par pressoir. Jus de goutte et jus de presse étaient ensuite assemblés et mis à fermenter dans des dolia dont les alignements forment l'équipement le plus caractéristique des chais. Les jarres étaient enterrées jusqu'à l'épaule dans des fosses carrées ou rondes soigneusement remblayées avec du sable ou de la terre. On ne trouve généralement que les fonds, mais lorsqu'ils sont suffisamment complets pour être jaugés, ils présentent des contenances élevées, situées entre 15 et $20 \mathrm{hl}{ }^{120}$ (fig. 39).

Comme le recommandent unanimement les agronomes antiques, ces vaisseaux étaient enduits de poix chaque année. Cette opération les rendait imperméables et évitait les fuites dans la terre. De plus, la nouvelle couverture de poix bouillante stérilisait l'intérieur des jarres et tuait les bactéries acétiques; le poissage jouait donc un rôle analogue au méchage au soufre. Dans quelques cas (à Pardigon 2, aux Toulons, à Saint-Martin), on a pu retrouver des amas de poix conservés au fond des dolia, mais généralement elle a disparu. En revanche, il arrive très fréquemment que les vases vinaires présentent un fond noirci par le feu. Ces traces ont été laissées par les feux que l'on allumait au fond des jarres afin d'en réchauffer la paroi de sorte que la poix bouillante puisse s'étaler et former un vernis ${ }^{121}$. Cette particularité semble caractéristique des vasa vinaria. En effet, les jarres à huile n'étant jamais poissées n'ont pas de raison de présenter de telles traces.

Lorsque la fermentation était achevée, dans l'hiver et au printemps, venait le temps de la vente, à moins que la récolte n'ait été auparavant vendue sur pied. I.e vin de. Narbonnaise orientale était certainement commercialisé en vrac dans les villes proches, mais aussi expédié par bateaux dans des amphores G. 2, G. 3, G. 4 et G. 5. On suit leur diffusion dans la vallée du Rhône, à Rome, dans le bassin occidental mais aussi oriental de la Méditerranée (Laubenheimer, 1989a). Alors que, dans certains cas, le négociant venait prendre livraison du vin au domaine avec ses amphores, il semble que bien souvent le vin n'ait pas été mis en amphore à la propriété. Prenons deux exemples. À Rians, le grand domaine des

120. En fait les contenances sont très variables. Dans la villa de La Condamine à Pontaix (Drôme), les dolia portaient des indications de capacité. L'inscription la plus complète : ur(nae) XXXV. b(issextia) III donne une capacité de 462,83 litres (Desaye, Blanc, 1969, p. 214). Au Molard à Donzère, les contenances variaient de 91 à 95 urnae (soit entre 1195 et 1247 litres). On connaît aussi de très grands dolia: celui du musée de Nîmes était susceptible de contenir 2064 litres, celui de la Bâtie-Rolland (Drôme), 2160 litres, celui d'Aubignosc (Alpes-deHaute-Provence), 1700 litres, celui de Vauclaire (Bouches-du-Rhône), 1870 litres, ceux de Rians (Var) entre 1000 et 1600 litres.

121. Sur cette question, voir les expériences réalisées à Beaucaire dans le Gard (Tchernia, Brun, 1999, p. 94-105). 
Toulons ne produisait pas d'amphores; il est vraisemblable, vu la proximité de la Durance, que le vin était acheminé dans des cullei placés sur des chariots jusqu'à la rivière sur les bords de laquelle il devait être conditionné. Dans la vallée de l'Argens, nombre de domaines (SaintMartin, L'Ormeau à Taradeau, Le Suveret et Roussivau à Saint-Raphaël, Villepey et Saint-Aygulf à Fréjus) semblent ne pas avoir possédé d'atelier de potier domanial. Tout se passe comme si le vin était enlevé en vrac par des négociants et mis en amphore au port de Fréjus où, ces vingt dernières années, plusieurs officines de potiers d'amphores ont été mises au jour (Le Pauvadou, SaintLambert, La Madeleine, $c f$. Gébara, Béraud, 1996). On aurait alors un fonctionnement proche de celui de l'expédition de l'huile en Bétique, transportée en vrac jusqu'aux ateliers situés en bordure du fleuve, au plus près des points d'embarcation (voir aussi le même phénomène sur l'étang de Thau, infra, p. 98).

\section{ESSOR ET VICISSITUDES DE LA VITICULTURE AU HAUT-EMPIRE}

Les résultats des recherches sur les installations vinicoles de Provence montrent une tendance générale au développement durant tout le Haut-Empire. Mais cet essor n'est pas allé sans vicissitudes individuelles, voire sans crises limitées à telle ou telle catégorie d'exploitation. De petits domaines ayant précocement pratiqué la viticulture comme Le Suveret et Tout-Égau ont été abandonnés très tôt, dès avant la fin du $\mathrm{I}^{\mathrm{cr}} \mathrm{s}$. après J.-C., soit que leur terroir, marginal, ait été rapidement épuisé, soit qu'ils aient été rachetés par des propriétaires voisins plus dynamiques. Ces quelques abandons, qui se produisent au moment où la viticulture est en plein essor, marquent peut-être une crise de croissance. Certaines petites exploitations, incapables de s'adapter, ont dû être marginalisées et sans doute absorbées par des unités plus grandes.

Sous les Flaviens et au début du II $^{\mathrm{e}} \mathrm{s}$., on assiste à la construction d'un grand nombre de chais : à L'Ormeau, à Forbin 1, à Pardigon 3, au Grand Loou, aux Mesclans, aux Toulons, à Tourville, à Notre-Dame-d'Avinionet (Mandelieu, Alpes-Maritimes, cf. Fixot dir., 1990), SaintChristophe (Biot, Alpes-Maritimes) ${ }^{122}$. Le mouvement

122. Voir Dumont, Violino, 1992. Il n'est pas certain que cette installation, qui comprend un pressoir et un cellier comptant 18 dolia, soit un atteint son apogée au milieu du II ${ }^{\mathrm{c}} \mathrm{s}$; c'est à cette époque que sont construits les chais de Saint-Martin, de Forbin 2, de Barresse et que celui du Grand Loou est agrandi.

Quelle que soit leur taille, tous ces équipements, bien construits (grands bâtiments, sols et cuves en béton, pressoirs puissants, nombreux dolia), ont nécessité des investissements importants. Il est significatif que même de petites exploitations comme celles de Berthoire, de L'Ormeau et de Barresse aient été équipées en grand. Grâce aux fouilles et aux prospections, on dispose désormais d'une base documentaire suffisante pour affirmer que, vers le milieu du $\mathrm{II}^{\mathrm{c}} \mathrm{s}$., quasiment toutes les exploitations de basse Provence produisaient du vin ou de l'huile et le plus souvent les deux (Brun, 1999, p. 152-154). La viticulture gagna aussi les Alpes : à l'installation viticole de La Bâtie-Montsaléon citée plus haut, il faut ajouter les cuves mises au jour à Saint-Ariès (Ventavon, Hautes-Alpes, cf. Guillaume, 1997), les dolia certainement vinaires de la villa de La Condamine à Pontaix (Drôme) ${ }^{123}$, ainsi que l'inscription mentionnant deux tiers d'arpents de vignes dans le jardin funéraire de Publicius Calistius à Die ${ }^{124}$.

Le cas de Barresse est probablement emblématique de la situation atteinte vers le milieu du II $^{\circ}$ s. L'implantation de cette petite ferme, et probablement de tout un village, dans les collines dominant la plaine du Muy, pourrait s'expliquer dans le cadre de la Lex Manciana et de la Lex Hadriana qui ont conféré aux paysans le droit d'occuper et de posséder à titre héréditaire les terres incultes à condition qu'ils les défrichent, les mettent en culture et qu'ils payent l'impôt. La longue phase de croissance économique et démographique du $\mathrm{I}^{\mathrm{er}} \mathrm{s}$. et du début du $\mathrm{II}^{\mathrm{e}} \mathrm{s}$. aurait produit un surpeuplement et la

chai à vin. En effet, à Peymeinade (Alpes-Maritimes), G. Vindry a dégagé plusieurs huileries bien identifiées par des moulins. Or les cuves de décantation et de stockage sont remplacées par des dolia enterrés.

123. Voir Blanc, Desaye, 1964, p. 272-275: le terrain de la villa de La Condamine fut défoncé en 1960. Une photographie aérienne prise alors montre qu'il s'agissait d'un vaste établissement couvrant 2 ha et organisé autour de cours à portiques. Le site, occupé à partir du I ${ }^{\mathrm{er}} \mathbf{s}$. avant J.-C., a livré de nombreux fragments de dolia dont trois portant des marques de contenance en urnae comme celles du Molard à Donzère.

124. CIL XII, $1657: D$ (iis) M(anibus) / Liberorum ac con/iugibus Publici Calis/ti et ipsius consecratum / cum bes(s)e vineae arep(ennis) / ex cuius reditu omnib / annis prolibari volo / ne minus XV v(ini) se(xtarii) / H(ic) $t$ (umulus) h(eredem) $n$ (on) s(equitur). 
nécessité de conquérir de nouvelles terres, marginales, où se serait répandu le trop-plein de population. Ces petits paysans auraient alors pratiqué une polyculture incluant la vigne et l'olivier, l'élevage de moutons dans les collines, ainsi qu'un petit artisanat servant à obtenir un peu de numéraire (dans le cas de Barresse, la production de céramiques de cuisine). Un phénomène similaire peut être observé dans les massifs collinaires bordant les plaines d'Antioche et de Chalcis en Syrie avec la construction de très nombreux villages de paysans libres (Tate, 1992, p. 298-299).

Dans le même temps, le mouvement de concentration de terres, dont on croit percevoir les prémices dès la fin du $\mathrm{I}^{\mathrm{er}} \mathrm{s}$., se poursuivit au cours du $\mathrm{II}^{\mathrm{e}} \mathrm{s}$. Deux phénomènes se manifestent à partir du milieu du siècle. Le premier est l'achat, par des propriétaires non résidents, de domaines où, jusqu'alors, vivaient des familles de notables. Ce pourrait être le cas de Saint-Martin (Taradeau) et de Forbin 2 (Aix-en-Provence). La villa de Saint-Martin fut occupée au I ${ }^{\text {cr }}$ s. par deux familles dont les noms sont connus. Résidant manifestement sur place, les propriétaires avaient fait aménager un jardin intéricur avec bassin, des salles de réception mosaiquées et des thermes; dans la seconde moitié du $\mathrm{II}^{\mathrm{e}}$ s., le domaine dut être vendu à un personnage qui, dans la pars urbana, installa des fouloirs, des pressoirs et des celliers. Il n'existait plus alors de lieu de résidence et on doit imaginer que le propriétaire disposait d'une villa voisine pour ses séjours ${ }^{125}$. Le cas de Forbin 2 paraît similaire à ceci près que le nouveau propriétaire habitait peut-être Aquae Sextiae distante de $1 \mathrm{~km}$ seulement.

À la fin du II ${ }^{\mathrm{e}} \mathrm{s}$. et au début du III ${ }^{\mathrm{e}} \mathrm{s}$., la concentration devient plus manifeste car elle conduisit à l'abandon de certaines villae. L'exemple le plus net est celui de la baie de Cavalaire : après l'abandon de la villa de Pardigon 3, ses ruines et sa carrière de pierre furent exploitées pour agrandir la villa voisine de Pardigon 2. Il paraît vraisemblable que les vignobles de la première furent regroupés avec ceux de la seconde (Congès, Lecacheur, 1994). La villa du Grand Loou 1 fut aussi abandonnée à la même époque tandis que la villa voisine du Grand Loou 3 resta occupée durant l'Antiquité tardive ; il est tentant de voir, là aussi, un regroupement de terres au profit de la seconde. Il en va peutêtre de même à Taradeau où le hameau

125. On pense bien sûr à la célèbre lettre de Pline lc Jeune sur l'opportunité d'arrondir ses terres en acquérant un domaine voisin (Epist., III, 19). agricole de L'Ormeau est abandonné vers la fin du II ${ }^{\mathrm{c}}$ s., au moment où l'exploitation viticole de Saint-Martin, distante de moins de $1 \mathrm{~km}$, fonctionne à plein rendement.

Toutefois, cette concentration progressive de terres, bien décrite dès la fin du ${ }^{\mathrm{er}} \mathrm{s}$. par Hygin ${ }^{126}$, n'affectait pas la productivité globale : les vignobles continuaient à être entretenus et on ne décèle aucune baisse de la diffusion des amphores gauloises dans la première moitié du III' $s$.

\section{VITICULTURE DURANT L'ANTIQUITÉ TARDIVE}

Appréhender la place de la viticulture au cours de l'Antiquité tardive est rendu difficile car l'utilisation grandissante des récipients et instruments en bois fausse notre perception. La progressive disparition des amphores gauloises au début $d u I^{c} \mathrm{~s}$. est-elle le reflet fidèle du recul de la viticulture commerciale ou bien seulement celui de leur remplacement par des tonneaux ? L'enlèvement des dolia ou leur comblement montrent-ils de même une chute de la production ou leur remplacement par des foudres ? Ou bien encore, les deux phénomènes se conjuguent-ils, mais dans quelles proportions? Les seuls points fermes sur lesquels on peut se fonder sont les datations d'abandon des villae et du comblement des cuves de recueil.

Les cas analysables à notre disposition montrent que les villae qui n'ont pas été abandonnées au III $\mathrm{s}$. ont continué d'être occupées jusqu'au Ve ${ }^{e}$ s. (Brun, Congès, 1996), mais rares sont celles dont on est sûr que l'exploitation vinicole a continué au cours de l'Antiquité tardive : Saint-Martin à Taradeau et peut-être Saint-Pierre à Eyguières (Bouches-du-Rhône) si le pressoir retrouvé correspond à une installation vinicole et non à une huilerie. Ailleurs, il est des cas où l'on est certain que la production de vin et d'huile a cessé dans la villa même

126. « Il arrive d'habitude que des propriétaires de plusieurs domaines contigus incorporent pour ainsi dire deux ou trois fonds de terre à une seule villa et laissent les bornes qui limitaient chaque fond de terre individuellement. Ayant abandonné les autres villae sauf celle à laquelle les fonds voisins ont été incorporés, et non satisfaits de leurs propres confins, ils enlèvent les bornes de lcurs voisins par lesquelles est limitée leur possession et défendent pour eux-mêmes les bornes par lesquelles les confins d'un seul propriétaire sont désormais respectés. " (Behrends et al., 2000, $§ 170$ ). 
(Les Mesclans et Notre-Dame-d'Avinionet à Mandelieu) et d'autres où c'est probable. Dans les villae des Toulons à Rians et de la rue du Port à Cavalaire-sur-Mer, les dolia furent cassés et enlevés, mais on ne peut exclure qu'ils aient été remplacés par des foudres.

La diminution du nombre et de la taille des installations en fonction au cours des $\mathrm{IV}^{\mathrm{e}}$ et $\mathrm{V}^{\mathrm{c}} \mathrm{s}$. signifie probablement un recul de la viticulture, mais il est certainement faux de penser que ce recul est proportionnel à celui du nombre d'installations. On sait bien que l'on continuait de faire du vin en Provence au cours de l'Antiquité tardive: Paulin rappelle que Marseille en tirait encore l'essentiel de ses ressources au $V^{e}$ s.; le testament d'Abbon indique qu'il y avait des vignes dans l'arrière-pays de Toulon en 739.

La situation semble donc complexe : quelques exploitations continuaient d'exploiter leurs vignes en fairevaloir direct et une part grandissante de domaines devait en concéder l'exploitation à des métayers (coloni). Le phénomène de concentration des terres, esquissé dès la fin du Irr s., s'accrut au IV $\mathrm{s}$. La villa cessa progressivement d'être un lieu de production pour devenir, outre la résidence du maître, un centre de gestion du domaine, un lieu de perception de la rente foncière en nature, un lieu de stockage des denrées apportées par les coloni. C'est ce modèle qui pourrait expliquer l'abandon des installations de production agricole alors que la pars urbana des villae continuait à être occupée. Cette hypothèse a été présentée pour expliquer l'abandon de la pars agraria de la villa des Mesclans à La Crau (Brun, Borréani, 1998).

Elle implique que le vin ait été produit en petites quantités par des métayers dans leurs fermes. Pour qu'elle soit validée, il faudrait donc que l'on parvienne à fouiller des habitations de coloni de l'Antiquité tardive, mais, là, on se heurte à trois obstacles. Premièrement, ces habitations, par définition peu importantcs, sont difficiles à déceler en prospection car elles ne laissent que peu de traces. Deuxièmement, étant petites et bâties en pierre sèche, elles sont facilement détruites par les labours, ce qui signifie que, lorsqu'on émet l'hypothèse de la découverte d'une telle habitation au vu du résultat des prospections, il y a toutes chances pour que le site soit déjà détruit. Troisièmement, si production de vin il y a eu dans de telles exploitations, elle ne devait pas atteindre un volume suffisant pour nécessiter des installations bâties susceptibles de laisser des vestiges inter- prétables ${ }^{127}$. Il faudrait plutôt imaginer des fouloirs et des cuves en bois, au mieux un pressoir à vis directe, et quelques tonneaux. Autant dire que les chances de mettre au jour des traces de vinification sont pratiquement nulles. On se trouverait donc dans une situation proche de celle des vestiges de l'âge du Fer où la viticulture est essentiellement attestée par les macrorestes.

Cette hypothèse n'explique tout de même pas l'ensemble du phénomène que l'on croit observer. Au IV $\mathrm{e}$., dans les provinces où les sources écrites mentionnent une viticulture florissante, comme en Aquitaine ou dans la vallée de la Moselle, les grands propriétaires continuent à produire leur vin eux-mêmes, au moins en partie, ne serait-ce que pour des raisons de prestige bien analysées par Whittaker (1985) ${ }^{128}$. Le thème du don de la vigne figuré sur une mosaïque de la villa de Vinon-surVerdon (Var) ne s'interprète correctement que si le dominus était un fier producteur de vin du début du $V^{\mathrm{e}} \mathrm{s}$. (Lavagne, 1994). Or force est de constater que, le cas de Vinon mis à part ${ }^{129}$, on n'a pas trouvé jusqu'ici de lien entre les villae tardives et la production de vin. Il faut probablement en conclure que la production vinicole à cette époque n'avait plus l'importance économique et sociale qui avait été la sienne au Haut-Empire.

On le voit, la rareté des études sur les habitats ruraux tardifs grands ou petits rend incertaine toute analyse de la viticulture en Provence durant l'Antiquité tardive. Celle-ci fait pâle figure après l'acmé du II ${ }^{\mathrm{e}} \mathrm{s}$. Les avancées sur ce sujet ne pourront venir que du dégagement total de grands domaines tardifs et de quelques habitations de petite taille, travaux qu'il faudra coupler avec des études carpologiques, palynologiques et anthracologiques.

127. Dans la ferme de L'Ormeau B à Taradeau (Var), les fouilles ont mis en évidence une habitation tardive aménagée dans les ruines de la ferme du Haut-Empire. Je me demande désormais s'il ne s'agit pas d'une habitation de colon dépendant de la villa voisine de Saint-Martin. Mais rien dans les découvertes ne peut être relié à une éventuelle production de vin à cette époque.

128. À la fin de la République et au début de l'Empire, les grands propriétaires mettaient leur fierté dans la course au rendement de leurs vignes plutôt que dans la qualité de leurs vins (Tchernia, 1995a). Mais dès cette époque, ils s'enorgueillissaient, comme Trimalcion dans le Satiricon, de pouvoir offrir du vin de toutes leurs propriétés. Durant l'Antiquité tardive, l'accent semble avoir été mis sur la qualité, et les évêques développeront cette tendance.

129. Rappelons toutefois qu'à Vinon seule la partie d'habitation a été partiellement fouillée et que l'on ne sait rien d'éventuelles installations vinicoles. 